\title{
The ultrafilter and almost disjointness numbers
}

\author{
Osvaldo Guzmán Damjan Kalajdzievski *
}

\begin{abstract}
We prove that every MAD family can be destroyed by a proper forcing that preserves $P$-points. With this result, we prove that it is consistent that $\omega_{1}=\mathfrak{u}<\mathfrak{a}$, solving a nearly 20 year old problem of Shelah, and a problem of Brendle. We will also present a simple proof of a result of Blass and Shelah that the inequality $\mathfrak{u}<\mathfrak{s}$ is consistent.
\end{abstract}

\section{Introduction}

Ultrafilters and MAD familie 1 play a fundamental role on infinite combinatorics, set theoretic topology and other branches of mathematics. For this reason, it is interesting to study the relationship between these two objects. In this paper, we will focus on the cardinal invariants associated with each of them. The ultrafilter number $\mathfrak{u}$ is defined as the smallest size of a base of an ultrafilter, and the almost disjointness number $\mathfrak{a}$ is the smallest size of a MAD family. The consistency of the inequality $\mathfrak{a}<\mathfrak{u}$ is well known and easy to prove, in fact, it holds in the Cohen, random, and Silver models, among many others. Proving the consistency of the inequality $\mathfrak{u}<\mathfrak{a}$ is much harder and used to be an open problem for a long time; In fact, it follows by the theorems of Hrušák, Moore and Džamonja that the inequality $\mathfrak{u}<\mathfrak{a}$ can not be obtained by using countable support iteration of proper Borel partial orders (see theorem 6.6 and theorem 7.2 of [52]). The consistency of $\mathfrak{u}<\mathfrak{a}$ was finally established by Shelah in 60] (see also [11]) where he proved the following theorem:

Theorem 1 (Shelah) Let $V$ be a model of $G C H, \kappa$ a measurable cardinal and $\mu, \lambda$ two regular cardinals such that $\kappa<\mu<\lambda$. There is a c.c.c. forcing extension of $V$ that satisfies $\mu=\mathfrak{b}=\mathfrak{d}=\mathfrak{u}$ and $\lambda=\mathfrak{a}=\mathfrak{c}$. In particular, CON (ZFC + "there is a measurable cardinal") implies $\operatorname{CON}(Z F C+" \mathfrak{u}<\mathfrak{a}$ ").

*The first author was partially supported by a CONACyT grant A1-S-16164 and PAPIIT grant IN104220. The second author was supported by York University.

keywords: MAD families, ultrafilters, almost disjointness number, P-points ultrafilter number. AMS classification: 03E17, 03E35, 03E05

${ }^{1}$ The reader may consult the next section for the definitions of the undefined concepts used in the introduction. 
This theorem was one of the first results proved using "template iterations", which is a very powerful method that has been very useful and has continued applications to this day (see for example, [9], [11, [10, [25, [27, [45]). In spite of the beauty of this result, it leaves open the following questions:

Problem 2 (Shelah [59]) Does CON(ZFC) imply $\operatorname{CON}(Z F C+" \mathfrak{u}<\mathfrak{a} ")$ ?

Problem 3 (Brendle [11]) Is it consistent that $\omega_{1}=\mathfrak{u}<\mathfrak{a}$ ?

In this paper, we will provide a positive answer to both questions, by proving (without appealing to large cardinals) that every MAD family can be destroyed by a proper forcing that preserves $P$-points. We will also present an alternative proof of the consistency of $\mathfrak{u}<\mathfrak{s}$, which was proved first by Blass and Shelah in [7] (see also [3]).

Our motivation comes from the theorems of Shelah that establishes that the statements " $\omega_{1}=\mathfrak{b}=\mathfrak{a}<\mathfrak{s}=\omega_{2}$ " and " $\omega_{1}=\mathfrak{b}<\mathfrak{a}=\mathfrak{s}=\omega_{2}$ " are consistent (see [57, or [58]). After these impressive results, different models of $\omega_{1}=\mathfrak{b}<\mathfrak{a}=\omega_{2}$ had been constructed (see for example [20, [12, 26] and [8]). In every case, the forcings used add Cohen reals, so no ultrafilter is preserved.

In order to construct the models of $\mathfrak{b}<\mathfrak{s}$ and $\mathfrak{b}<\mathfrak{a}$, Shelah used a creature forcing (see [57, [58, and [2]). In [15, Brendle and Raghavan found a simpler representation of Shelah forcing as a two step iteration, which we will briefly describe (more details of the forcing will be studied in the next section).

The most natural way to increase the splitting number is to diagonalize an ultrafilter. In order to build a model of $\mathfrak{b}<\mathfrak{s}$, it is enough to construct (or force) an ultrafilter that can be diagonalized without adding dominating reals (even in the iteration). Denote by $\mathbb{F}_{\sigma}$ the set of all $F_{\sigma}$-filter $2^{2}$ on $\omega$. If $\mathcal{F}, \mathcal{G} \in \mathbb{F}_{\sigma}$ we define $\mathcal{F} \leq \mathcal{G}$ if $\mathcal{G} \subseteq \mathcal{F}$. It is not hard to see that $\mathbb{F}_{\sigma}$ naturally adds an ultrafilter $\mathcal{U}_{\text {gen }}$. The reader wishing to learn more about $\mathbb{F}_{\sigma}$ may consult [41] and [49. It turns out that the forcing of Shelah is equivalent to the two step iteration $\mathbb{F}_{\sigma} * \mathbb{M}\left(\dot{\mathcal{U}}_{\text {gen }}\right)$, where $\mathbb{M}\left(\mathcal{U}_{\text {gen }}\right)$ is the Mathias forcing relative to $\mathcal{U}_{\text {gen }}$ (see [15]). It can be proved that $\mathbb{M}\left(\mathcal{U}_{\text {gen }}\right)$ does not add dominating reals, even when iterated (see [15] or [28]).

The method to build a model of $\mathfrak{b}<\mathfrak{a}$ is similar: Given a MAD family $\mathcal{A}$, denote by $\mathbb{F}_{\sigma}(\mathcal{A})$ the set of all $F_{\sigma}$-filters $\mathcal{F}$ such that $\mathcal{F} \cap \mathcal{I}(\mathcal{A})=\emptyset$ (where $\mathcal{I}(\mathcal{A})$ is the ideal generated by $\mathcal{A})$. Once again, we order $\mathbb{F}_{\sigma}(\mathcal{A})$ with inclusion. It is easy to see that $\mathbb{F}_{\sigma}(\mathcal{A})$ naturally adds an ultrafilter $\mathcal{U}_{\text {gen }}(\mathcal{A})$, furthermore, diagonalizing $\dot{\mathcal{U}}_{\text {gen }}(\mathcal{A})$ destroys the maximality of $\mathcal{A}$. In this case, it can be proved that $\mathbb{C}_{\omega_{1}} * \mathbb{F}_{\sigma} * \mathbb{M}\left(\dot{\mathcal{U}}_{\text {gen }}(\mathcal{A})\right)$ does not add dominating reals, even when

\footnotetext{
${ }^{2}$ We view filters as subspaces of $2^{\omega}$, the notion of Borel or $F_{\sigma}$ is taken using the usual topology on $2^{\omega}$.
} 
iterated $\left(\mathbb{C}_{\omega_{1}}\right.$ denotes the forcing for adding $\omega_{1}$-Cohen reals), see [15] or [28]. We want to point out that both the forcing of Shelah $([57,[58])$ and the forcing used by Brendle in 8 r require adding Cohen reals in an explicit way. Our work shows that adding the Cohen reals was in fact not needed.

We take a similar approach in order to build our model of $\mathfrak{u}<\mathfrak{s}$. We will first force with $\mathbb{F}_{\sigma}$ and then we will diagonalize $\dot{\mathcal{U}}_{g e n}$. The difference, is that instead of using Mathias forcing, we will use a variant of Miller forcing. The same technique will be used to build the model of $\omega_{1}=\mathfrak{u}<\mathfrak{a}$. Given a MAD family $\mathcal{A}$, we will force with $\mathbb{F}_{\sigma}(\mathcal{A})$ and then diagonalize in the same way as before. In both cases, we will prove that the forcings preserve all $P$-points of the ground model.

There is a huge body of work regarding the cardinal invariants $\mathfrak{b}, \mathfrak{s}, \mathfrak{d}, \mathfrak{u}$, and $\mathfrak{a}$; To provide some more historical context to this work, as was mentioned before, the story began when Shelah ([58]) constructed models of $\omega_{1}=\mathfrak{b}<\mathfrak{s}$ and $\omega_{1}=\mathfrak{b}<\mathfrak{a}=\mathfrak{s}$. To achieve this, Shelah used a countable support iteration of creature forcings. In [8], Brendle used c.c.c. forcings for constructing models of $\kappa=\mathfrak{b}<\mathfrak{a}=\kappa^{+}$, where $\kappa$ is any uncountable regular cardinal, and later in [26] Fischer and Steprāns constructed models of $\kappa=\mathfrak{b}<\mathfrak{s}=\kappa^{+}$, where again $\kappa$ can be any uncountable regular cardinal. In 12 Brendle and Fischer used matrix iterations to prove that for any regular cardinals $\kappa<\lambda$, it is consistent that $\kappa=\mathfrak{b}=\mathfrak{a}<\mathfrak{s}=\lambda$, and if $\kappa$ is bigger than a measurable cardinal, then it is consistent that $\kappa=\mathfrak{b}<\mathfrak{a}=\mathfrak{s}=\lambda$. The consistency of $\omega_{1}<\mathfrak{d}<\mathfrak{a}$ and $\omega_{1}<\mathfrak{u}<\mathfrak{a}$ was obtained by Shelah in $[60$, where he developed the technique of forcing along a template (see also [9] and [11). Finally, in [25] Fischer and Mejía proved that it is consistent that $\omega_{1}<\mathfrak{s}<\mathfrak{b}<\mathfrak{a}$ (see also [46] and [23]).

There are still many interesting open questions remaining:

Problem 4 (Roitman) Does $\mathfrak{d}=\omega_{1}$ imply that $\mathfrak{a}=\omega_{1}$ ?

Problem 5 (Brendle and Raghavan) Does $\mathfrak{b}=\mathfrak{s}=\omega_{1}$ imply that $\mathfrak{a}=\omega_{1}$ ?

Note that a positive solution to the question of Brendle and Raghavan would provide a positive solution to the problem of Roitman.

\section{Preliminaries and notation}

Let $f, g \in \omega^{\omega}$, define $f \leq g$ if and only if $f(n) \leq g(n)$ for every $n \in \omega$ and $f \leq^{*} g$ if and only if $f(n) \leq g(n)$ holds for all $n \in \omega$ except finitely many. We say a family $\mathcal{B} \subseteq \omega^{\omega}$ is unbounded if $\mathcal{B}$ is unbounded with respect to $\leq^{*}$. A family $\mathcal{D} \subseteq \omega^{\omega}$ is a dominating family if for every $f \in \omega^{\omega}$, there is $g \in \mathcal{D}$ such that $f \leq^{*} g$. The bounding number $\mathfrak{b}$ is the size of the smallest unbounded family and the dominating number $\mathfrak{d}$ is the smallest size of a dominating family. 
We say that $S$ splits $X$ if $S \cap X$ and $X \backslash S$ are both infinite. A family $\mathcal{S} \subseteq$ $[\omega]^{\omega}$ is a splitting family if for every $X \in[\omega]^{\omega}$ there is $S \in \mathcal{S}$ such that $S$ splits $X$. The splitting number $\mathfrak{s}$ is the smallest size of a splitting family. A family $\mathcal{A} \subseteq[\omega]^{\omega}$ is almost disjoint $(A D)$ if the intersection of any two different elements of $\mathcal{A}$ is finite, a MAD family is a maximal almost disjoint family. $\operatorname{By} \operatorname{cov}(\mathcal{M})$ we denote the smallest size of a family of meager sets that covers the Baire space. The reader may consult the surveys [5] for the main properties of the cardinal invariants used in this paper, 33 to learn more about almost disjoint families and 32 for a survey on filters and ideals.

In this paper, a tree is a set of sequences closed under taking restrictions (i.e. $p$ is a tree if whenever $s \in p$ and $n<|s|$ then $s\left\lceil n \in p\right.$ ). If $s, t \in \omega^{<\omega}$, by $s \frown t$ we denote the concatenation of $s$ and $t$. If $n \in \omega$, we will often write $s \frown n$ instead of $s^{\frown}\langle n\rangle$. In this paper, we will say that $s \in p$ is a splitting node if $\operatorname{suc}_{p}(s)=\left\{n \mid s^{\frown} n \in p\right\}$ is infinite. We say that a splitting node $s \in p$ is the stem of $p$ (denoted by stem $(p)$ in case it exists) if every predecessor of $s$ has exactly one immediate successor. If $p$ is a tree, the set of branches of $p$ is defined as $[p]=\{x \mid \forall n(x\lceil n \in p)\}$. For every $s \in p$, we define the tree $p_{s}=\{t \in p \mid s \subseteq t \vee t \subseteq s\}$. Given $s \in \omega^{<\omega}$ define the cone of $s$ as $\langle s\rangle=$ $\left\{f \in \omega^{\omega} \mid s \subseteq f\right\}$.

Let $\mathcal{I}$ be an ideal on $\omega, \mathcal{F}$ a filter on $\omega$ and $\mathcal{A}$ a MAD family. Define 3 $\mathcal{I}^{+}=\wp(\omega) \backslash \mathcal{I}$ i.e. the subsets of $\omega$ that are not in $\mathcal{I}$. We say that a forcing notion $\mathbb{P}$ destroys $\mathcal{I}$ if $\mathbb{P}$ adds an infinite subset of $\omega$ that is almost disjoint with every element of $\mathcal{I}$. We say that $\mathbb{P}$ diagonalizes $\mathcal{F}$ if $\mathbb{P}$ adds an infinite set almost contained in every element of $\mathcal{F}$. It is easy to see that $\mathbb{P}$ destroys $\mathcal{I}$ if and only if $\mathbb{P}$ diagonalizes the filter $\mathcal{I}^{*}=\{\omega \backslash A \mid A \in \mathcal{I}\}$. By $\mathcal{I}(\mathcal{A})$ we denote the ideal generated by $\mathcal{A}$ (and the finite sets). We say that $\mathbb{P}$ destroys $\mathcal{A}$ if $\mathcal{A}$ is no longer maximal after forcing with $\mathbb{P}$. Note that $\mathbb{P}$ destroys $\mathcal{A}$ if and only if $\mathbb{P}$ destroys the ideal $\mathcal{I}(\mathcal{A})$. The following result is well known (as well as easy to prove) and will be frequently used:

Lemma 6 Let $\mathcal{A}$ be a MAD family. If $B \subseteq \omega$, the following are equivalent:

1. $B \in \mathcal{I}(\mathcal{A})^{+}$.

2. There is $\mathcal{A}_{1} \in[\mathcal{A}]^{\omega}$ such that $|B \cap A|=\omega$ for every $A \in \mathcal{A}_{1}$.

We will need to recall the definition of the Katětov order:

Definition 7 Let $A$ and $B$ be two countable sets, $\mathcal{I}, \mathcal{J}$ be ideals on $X$ and $Y$ respectively and $f: Y \longrightarrow X$.

1. We say that $f$ is a Katětov morphism from $(Y, \mathcal{J})$ to $(X, \mathcal{I})$ if $f^{-1}(A) \in \mathcal{J}$ for every $A \in \mathcal{I}$.

\footnotetext{
${ }^{3} \mathrm{By} \wp(a)$ we denote the powerset of $a$.
} 
2. We define $\mathcal{I} \leq_{\mathrm{k}} \mathcal{J}(\mathcal{I}$ is Katětov smaller that $\mathcal{J}$ or $\mathcal{J}$ is Katětov above $\mathcal{I})$ if there is a Katětov morphism from $(Y, \mathcal{J})$ to $(X, \mathcal{I})$.

The reader may consult [34] for an interesting survey of the Katětov order on Borel ideals. The Katětov order does not play a crucial result in this article, but we include it in order to state some results of Sabok and Zapletal below. The nowhere dense ideal nwd is the ideal on $2^{<\omega}$ where $A \in$ nwd if and only if for every $s \in 2^{<\omega}$ there is $t \in 2^{<\omega}$ extending $s$ such that no further extension of $t$ is in $A$. It is easy to see that nwd is an ideal. For every $n \in \omega$ we define $C_{n}=\{(n, m) \mid m \in \omega\}$ and if $f: \omega \longrightarrow \omega$ let $D(f)=\{(n, m) \mid m \leq f(n)\}$. The ideal fin $\times$ fin is the ideal on $\omega \times \omega$ generated by $\left\{C_{n} \mid n \in \omega\right\} \cup\left\{D(f) \mid f \in \omega^{\omega}\right\}$.

If $\mathcal{F}$ is a filter on $\omega$ (or on any countable set) we define the Mathias forcing $\mathbb{M}(\mathcal{F})$ with respect to $\mathcal{F}$ as the set of all pairs $(s, A)$ where $s \in[\omega]^{<\omega}$ and $A \in \mathcal{F}$. If $(s, A),(t, B) \in \mathbb{M}(\mathcal{F})$ then $(s, A) \leq(t, B)$ if the following conditions hold:

1. $t$ is an initial segment of $s$.

2. $A \subseteq B$.

3. $(s \backslash t) \subseteq B$.

The Laver forcing $\mathbb{L}(\mathcal{F})$ with respect to $\mathcal{F}$ is the set of all trees $p$ such that $\operatorname{suc}_{p}(s) \in \mathcal{F}$ for every $s \in p$ extending the stem of $p$. We say $p \leq q$ if $p \subseteq q$.

While $\mathbb{L}(\mathcal{F})$ always adds a dominating real, this may not be the case for $\mathbb{M}(\mathcal{F})$. A trivial example is taking $\mathcal{F}$ to be the cofinite filter in $\omega$, since in this case $\mathbb{M}(\mathcal{F})$ is forcing equivalent to Cohen forcing. A more interesting example was found by Canjar in [18, where an ultrafilter whose Mathias forcing does not add dominating reals was constructed under $\mathfrak{d}=\mathfrak{c}$ (see also 29]). We say that a filter $\mathcal{F}$ is Canjar if $\mathbb{M}(\mathcal{F})$ does not add dominating reals. Let $\mathcal{F}$ be a filter on $\omega$. We define the filter $\mathcal{F}^{<\omega}$ on $[\omega]^{<\omega} \backslash\{\emptyset\}$ generated by $\left\{[A]^{<\omega} \backslash\{\emptyset\} \mid A \in \mathcal{F}\right\}$. Note that $X \in\left(\mathcal{F}^{<\omega}\right)^{+}$if and only if for every $A \in \mathcal{F}$, there is $s \in X$ such that $s \subseteq A$. It is important to emphasize that if $X \in\left(\mathcal{F}^{<\omega}\right)^{+}$then by convention $\emptyset \notin$ $X$ (recall that $\mathcal{F}^{<\omega}$ is a filter on $[\omega]^{<\omega} \backslash\{\emptyset\}$ ). In [36] Hrušák and Minami showed that the forcing properties of $\mathbb{M}(\mathcal{F})$ are closely related to the combinatorics of $\mathcal{F}^{<\omega}$. They proved the following result:

Proposition 8 ([36]) Let $\mathcal{F}$ be a filter on $\omega$. The following are equivalent:

1. $\mathcal{F}$ is Canjar.

2. For every $\left\{X_{n} \mid n \in \omega\right\} \subseteq\left(\mathcal{F}^{<\omega}\right)^{+}$there are $Y_{n} \in\left[X_{n}\right]^{<\omega}$ such that $\bigcup_{n \in \omega} Y_{n} \in\left(\mathcal{F}^{<\omega}\right)^{+}$. 
In 19 it was proved that a filter is Canjar if and only if is has the Menger property (as a subspace of $\wp(\omega)$ ). Canjar filters have been further studied in [6], 29, 28], 24] and 31. holds:

We will say that a family of functions $\mathcal{B} \subseteq \omega^{\omega}$ is a $\mathfrak{b}$-family if the following

1. Every element of $\mathcal{B}$ is an increasing function.

2. Given $\left\{f_{n} \mid n \in \omega\right\} \subseteq \mathcal{B}$ there is $g \in \mathcal{B}$ such that $f_{n} \leq^{*} g$ for every $n \in \omega$.

3. $\mathcal{B}$ is unbounded.

An example of a $\mathfrak{b}$-family would be a well-ordered unbounded family, another example is the set of all increasing functions. If $\mathcal{B}$ is a $\mathfrak{b}$-family and $\mathbb{P}$ is a partial order, we say that $\mathbb{P}$ preserves $\mathcal{B}$ if $\mathcal{B}$ is still unbounded after forcing with $\mathbb{P}$. Note that if $\mathbb{P}$ is a proper forcing that preserves $\mathcal{B}$, then $\mathcal{B}$ is still a $\mathfrak{b}$-family in the extension. We will need the following easy lemma:

Lemma 9 Let $\mathcal{B}$ be a $\mathfrak{b}$-family. If $\mathcal{B}=\bigcup_{n \in \omega} \mathcal{B}_{n}$, then there is $n \in \omega$ such that $\mathcal{B}_{n}$ is cofinal in $\mathcal{B}$ (i.e. for every $f \in \mathcal{B}$ there is $g \in \mathcal{B}_{n}$ such that $f \leq^{*} g$ ).

Proof. We will argue a proof by contradiction. Assume this is not the case, so for every $n \in \omega$ there is $f_{n} \in \mathcal{B}$ such that $f_{n}$ is not bounded by any element of $\mathcal{B}_{n}$. Since $\mathcal{B}$ is a $\mathfrak{b}$-family, we can find $g \in \mathcal{B}$ such that $f_{n} \leq^{*} g$ for every $n \in \omega$. Since $\mathcal{B}=\bigcup_{n \in \omega} \mathcal{B}_{n}$, there must be $m \in \omega$ such that $g \in \mathcal{B}_{m}$, hence $f_{m} \leq^{*} g$ which is a contradiction.

Given a sequence $\bar{X}=\left\{X_{n} \mid n \in \omega\right\} \subseteq[\omega]^{<\omega} \backslash\{\emptyset\}$ and $f \in \omega^{\omega}$, we define the set $\bar{X}_{f}=\bigcup_{n \in \omega}\left(X_{n} \cap \wp(f(n))\right)$. Note that $\mathcal{F}$ is Canjar if for every sequence $\bar{X}=\left\{X_{n} \mid n \in \omega\right\} \subseteq\left(\mathcal{F}^{<\omega}\right)^{+}$there is $f \in \omega^{\omega}$ such that $\bar{X}_{f} \in\left(\mathcal{F}^{<\omega}\right)^{+}$. Recall that by a theorem of Hrušák and Minami (see [36]), a filter $\mathcal{F}$ is Canjar if and only if $\mathbb{M}(\mathcal{F})$ does not add dominating reals. If $\mathcal{B}$ is a $\mathfrak{b}$-family, we say that $\mathcal{F}$ is $\mathcal{B}$-Canjar if for every sequence $\bar{X}=\left\{X_{n} \mid n \in \omega\right\} \subseteq\left(\mathcal{F}^{<\omega}\right)^{+}$there is $f \in \mathcal{B}$ such that $\bar{X}_{f} \in\left(\mathcal{F}^{<\omega}\right)^{+}$(such $\bar{X}_{f}$ is called a pseudointersection according to $\left.\mathcal{B}\right)$. Note that if $\mathcal{F}$ is $\mathcal{B}$-Canjar (for some $\mathfrak{b}$-family $\mathcal{B}$ ), then $\mathcal{F}$ is Canjar. As expected, $\mathcal{B}$ Canjar filters have a similar characterization as the one of Canjar. The following is a slight strengthening of proposition 1 of [28, which is a generalization of the theorem of Hrušák and Minami:

Proposition 10 Let $\mathcal{B}$ be a $\mathfrak{b}$-family. A filter $\mathcal{F}$ is a $\mathcal{B}$-Canjar filter if and only if $\mathbb{M}(\mathcal{F})$ preserves $\mathcal{B}$.

Proof. Assume that $\mathcal{F}$ does not preserve $\mathcal{B}$, in other words, there is a name $\dot{g}$ for an increasing function such that $1_{\mathbb{M}(\mathcal{F})} \Vdash$ " $\dot{g}$ is an upper bound for $\mathcal{B}$ ". For 
every function $f \in \mathcal{B}$ let $s_{f} \in[\omega]^{<\omega}, n_{f} \in \omega$ and $F_{f} \in \mathcal{F}$ such that $\left(s_{f}, F_{f}\right) \Vdash$ " $\forall i \geq n_{f}\left(f(i)<_{n_{f}} \dot{g}(i)\right)$ ". Since $\mathcal{B}$ is a $\mathfrak{b}$-family, there are $s \in[\omega]^{<\omega}, n \in \omega$ and a cofinal family $\mathcal{B}^{\prime} \subseteq \mathcal{B}$ such that $s_{f}=s$ and $n_{f}=n$ for every $f \in \mathcal{B}^{\prime}$.

For every $m \in \omega$ let $X_{m}$ be the set of all $t \in[\omega \backslash \bigcup s]^{<\omega}$ such that there is $F \in$ $\mathcal{F}$ with the property that $(s \cup t, F)$ decides $\langle\dot{g}(0), \ldots, \dot{g}(m)\rangle$ and $(s \cup t, F) \Vdash$ $" \dot{g}(m)<\max (t) "$. It is easy to see that $\bar{X}=\left\{X_{m} \mid m \in \omega\right\}$ is a sequence of sets in $\left(\mathcal{F}^{<\omega}\right)^{+}$. We will see that it has no pseudointersection according to $\mathcal{B}$. Since $\mathcal{B}^{\prime}$ is cofinal in $\mathcal{B}$, it is enough to show that $\bar{X}$ has no pseudointersection according to $\mathcal{B}^{\prime}$.

Aiming for a contradiction, assume that there is $f \in \mathcal{B}^{\prime}$ such that $\bar{X}_{f}$ is positive. Since $\bar{X}_{f} \cap\left[F_{f}\right]^{<\omega}$ is infinite, pick $t \in \bar{X}_{f} \cap\left[F_{f}\right]^{<\omega}$ such that $t \in$ $X_{k} \cap \wp(f(k))$ with $k>n$. Since $t \in X_{k}$ there is $F \in \mathcal{F}$ such that $(s \cup t, F) \Vdash$ $" \dot{g}(k) \leq \max (t) "$ and note that $(s \cup t, F) \Vdash " \dot{g}(k) \leq f(k) "$. In this way, $\left(s \cup t, F_{h} \cap F\right)$ forces both " $f(k)<\dot{g}(k)$ " and " $\dot{g}(k) \leq f(k)$ ", which is a contradiction.

Now assume that $\mathbb{M}(\mathcal{F})$ preserves $\mathcal{B}$. Let $\bar{X}=\left\langle X_{n} \mid n \in \omega\right\rangle$ be a sequence of sets in $\left(\mathcal{F}^{<\omega}\right)^{+}$. Let $r_{\text {gen }}$ be a $(V, \mathbb{M}(\mathcal{F}))$-generic real, observe that $\left[r_{\text {gen }}\right]^{<\omega}$ intersect infinitely every member of $\left(\mathcal{F}^{<\omega}\right)^{+}$. In this way, in $V\left[r_{\text {gen }}\right]$ we may define an increasing function $g: \omega \longrightarrow \omega$ such that $\left(r_{g e n} \backslash n\right) \cap g(n)$ contains a member of $X_{n}$. Since $\mathcal{F}$ preserves $\mathcal{B}$, then there is $f \in \mathcal{B}$ such that $f \not^{*} g$, we will see that $\bar{X}_{f}$ is positive. Let $F \in \mathcal{F}$ we must prove that $\bar{X}_{f} \cap[F]^{<\omega}$ is not empty. Since $F \in \mathcal{F}$, we know that $r_{g e n} \subseteq^{*} F$ so there is $k \in \omega$ such that $g(k)<f(k)$ and $r_{g e n} \backslash k \subseteq F$ and hence $\bar{X}_{f} \cap[F]^{<\omega} \neq \emptyset$.

Moreover, Canjar filters satisfy the following stronger property:

Lemma 11 Let $\mathcal{B}$ be a $\mathfrak{b}$-family and $\mathcal{F}$ a $\mathcal{B}$-Canjar filter. For every family $\bar{X}=\left\{X_{n} \mid n \in \omega\right\} \subseteq\left(\mathcal{F}^{<\omega}\right)^{+}$there is $f \in \mathcal{B}$ such that for every $n \in \omega$, if $Y_{n}=$ $\left\{s \in X_{n} \mid s \subseteq[f(n-1), f(n))\right\}$ (where $f(-1)=0$ ) then $\bigcup_{n \in \omega} Y_{n} \in\left(\mathcal{F}^{<\omega}\right)^{+}$.

Proof. The idea of the proof is similar to the previous one. Let $r_{g e n}$ be a $(\mathbb{M}(\mathcal{F}), V)$-generic real. In $V\left[r_{\text {gen }}\right]$ we find an increasing function $g \in \omega^{\omega}$ such that for every $n \in \omega$, the following holds: $r_{g e n} \cap[g(n-1), g(n))$ contains an element of $X_{n}$ (where $g(-1)=0$ ). Furthermore, we may assume that $g$ is unbounded over $V$ (this is possible since $\mathbb{M}(\mathcal{F})$ adds an unbounded rea 4 ). Since $\mathcal{F}$ is $\mathcal{B}$-Canjar, we can find $(s, A) \in \mathbb{M}(\mathcal{F})$ and $f \in \mathcal{B}$ such that $(s, A) \Vdash$ " $f \mathbb{Z}^{*} \dot{g}$ ". We claim that $f$ is the function we are looking for. Define $Y_{n}=$ $\left\{s \in X_{n} \mid s \subseteq[f(n-1), f(n))\right\}$ (for every $n \in \omega$ ) and $Y=\bigcup_{n \in \omega} Y_{n}$, we must prove that $Y \in\left(\mathcal{F}^{<\omega}\right)^{+}$. Let $B \in \mathcal{F}$, we must show that $Y$ contains an element of $B$. Without loss of generality, we may assume that $B \subseteq A$. We can now find $m \in \omega, t \in[\omega]^{<\omega}$ and $C \in \mathcal{F}$ such that the following holds:

1. $\max (s)<m, C \subseteq B, t \subseteq B$.

\footnotetext{
${ }^{4}$ It is well known that every $\sigma$-centered forcing adds an unbounded real.
} 
2. $\max (s)<\min (t)$.

3. $(s \cup t, C) \leq(s, B)$.

4. There is $w \in X_{m}$ such that $(s \cup t, C) \Vdash " w \subseteq[\dot{g}(m-1), \dot{g}(m))$ ".

5. $(s \cup t, C) \Vdash "(f(m-1) \leq \dot{g}(m-1))$ " and $(s \cup t, C) \Vdash "(\dot{g}(m)<f(m))$ ".

Such $m, t$ and $C$ can be obtained since $(s, B) \leq(s, A)$, so $(s, B)$ forces that $\dot{g}$ is an unbounded real that does not dominate $f$. Note that $w \subseteq t$, so $w \subseteq B$ and $(s \cup t, C) \Vdash$ " $[\dot{g}(m-1), \dot{g}(m)) \subseteq[f(m-1), f(m))$ ", which implies that $w \in Y_{m}$. This finishes the proof.

\section{$3 \quad$ Miller forcing based on filters}

The theory of destructibility of ideals is very important in forcing theory, since many important forcing properties may be stated in these terms. For example, it is well known that a forcing $\mathbb{P}$ adds a dominating real if and only if $\mathbb{P}$ destroys fin $\times$ fin. The reader may consult [35, [16, [40, 37] or [42 for more on destructibility of ideals.

In order to build models where $\mathfrak{a}$ is big and some other cardinal invariant is small, we need to be able to destroy a MAD family by dealing the least "damage" as possible to the ground model. The most well known forcings to destroy an ideal (or to diagonalize a filter) are the Mathias or Laver forcings relative to the ideal (filter). The following result is well known:

Proposition 12 Let $\mathcal{F}$ be a filter on $\omega$.

1. $\mathbb{L}(\mathcal{F})$ adds a dominating real.

2. $\mathbb{M}(\mathcal{F})$ adds a Cohen real if and only if $\mathcal{F}$ is not a Ramsey ultrafilter.

3. If $\mathcal{F}$ is a Ramsey ultrafilter, then $\mathbb{M}(\mathcal{F})$ adds a dominating real.

In particular, it follows that $\mathbb{M}(\mathcal{F})$ adds either a Cohen or a dominating real. In this section, we will introduce a forcing relative to a filter $\mathcal{F}$ that in some cases, might destroy $\mathcal{F}$ without adding Cohen or dominating reals.

We say that a tree $p \subseteq \omega^{<\omega}$ is a Miller tree if the following conditions hold:

1. $p$ consists of increasing sequences.

2. $p$ has a stem. 
3. For every $s \in p$, there is $t \in p$ such that $s \subseteq t$ and $t$ is a splitting node 5

Usually, Miller trees are required to satisfy the following extra condition:

4. Every node of $p$ either is a splitting node or it has exactly one immediate successor.

However, we will not assume this extra requirement. The Miller forcing $\mathbb{P T}$ consists of all Miller trees ordered by inclusion 6 Miller forcing (also called "super perfect forcing") was introduced by Miller in [50, and is one of the most useful and studied forcings for adding new reals (see [3], [50] or [30]). By split $(p)$ we denote the collection of all splitting nodes, and by $\operatorname{split}_{n}(p)$ we denote the collection of $n$-splitting nodes (i.e. $s \in \operatorname{split}_{n}(p)$ if $s \in \operatorname{split}(p)$ and $s$ has exactly $n$-restrictions that are splitting nodes). Note that $\operatorname{split}_{0}(p)=\{\operatorname{stem}(p)\}$.

In [55, Sabok and Zapletal introduced the following parametrized version of Miller forcing7:

Definition 13 Let $\mathcal{F}$ be a filter. By $\mathbb{Q}(\mathcal{F})$ we denote the set of all Miller trees $p \in \mathbb{P} \mathbb{T}$ such that suc $_{p}(s) \in \mathcal{F}^{+}$for every splitting node s. We order $\mathbb{Q}(\mathcal{F})$ by inclusion.

Sabok and Zapletal proved some very interesting results, like the following: (the reader may consult [55] and [47] for the definitions of spl and the Solecki ideal $\mathcal{S})$.

Proposition $14([55])$ Let $\mathcal{F}$ be a filter.

1. $\mathbb{Q}(\mathcal{F})$ does not add Cohen reals if and only if nwd $\mathbb{K}_{\mathrm{K}} \mathcal{F}^{*} \uparrow A$ for every $A \in \mathcal{F}^{+}$.

2. If $\mathcal{F}$ is a Borel filter, then $\mathbb{Q}(\mathcal{F})$ preserves outer Lebesgue measure if and only if $\mathcal{S} \not_{\mathrm{K}} \mathcal{F}^{*} \mid A$ for every $A \in \mathcal{F}^{+}$.

3. If $\mathcal{F}$ is a Borel filter, then $\mathbb{Q}(\mathcal{F})$ does not add splitting reals if and only if spl $\Varangle_{\mathrm{K}} \mathcal{F}^{*} \uparrow A$ for every $A \in \mathcal{F}^{+}$.

In some cases, $\mathbb{Q}(\mathcal{F})$ may diagonalize $\mathcal{F}$ while in some others cases not, as can be seen with the following:

\section{Lemma 15}

\footnotetext{
${ }^{5}$ Recall that $s$ is a splitting node of $p$ if $s u c_{p}(s)$ is infinite.

${ }^{6}$ Obviously, the trees satisfying property 4 are dense in Miller forcing. However, this does not seem to be the case for our forcings.

${ }^{7}$ In 55 the authors use ideals instead of filters. Evidently, this choice is superflous.
} 
1. $\mathbb{Q}\left(\right.$ fin $\times$ fin $\left.{ }^{*}\right)$ does not destroy fin $\times$ fin.

2. $\mathbb{Q}\left(n w d^{*}\right)$ destroys $n w d$.

Proof. With an easy fusion argument, it is possible to prove that $\mathbb{Q}(\mathcal{F})$ does not add dominating reals (for every $\mathcal{F}$ ). Alternatively, this can be proved as follows: In [55] it was proved that there is a $\sigma$-ideal $\mathcal{J}_{\mathcal{F}}$ generated by closed sets such that $\mathbb{Q}(\mathcal{F})$ is forcing equivalent to $\operatorname{Borel}\left(\omega^{\omega}\right) / \mathcal{J}_{\mathcal{F}}$, which does not add dominating reals by Theorem 4.1.2 of 62. It is well known that if a forcing destroys fin $\times$ fin, then it must add a dominating real. From these two facts, it follows that $\mathbb{Q}($ fin $\times$ fin* $)$ does not destroy fin $\times$ fin. On the other hand, note that $\mathbb{Q}\left(n w d^{*}\right)$ adds a Cohen real and it is easy to see that any forcing adding a Cohen real must destroy nwd.

We will now introduce a version of Miller forcing parametrized with a filter $\mathcal{F}$ that will always diagonalizes $\mathcal{F}$. Given $p \in \mathbb{P T}$ for every $s \in \operatorname{split}_{n}(p)$ we define $\operatorname{spsuc}_{p}(s)=\left\{t \backslash s \mid t \in \operatorname{split}_{n+1}(p) \wedge s \subseteq t\right\}$.

Definition 16 Let $\mathcal{F}$ be a filter. We say $p \in \mathbb{P} \mathbb{T}(\mathcal{F})$ if the following holds:

1. $p \in \mathbb{P} \mathbb{T}$.

2. If $s \in \operatorname{split}(p)$ then $\operatorname{spsuc}_{p}(s) \in\left(\mathcal{F}^{<\omega}\right)^{+}$.

We order $\mathbb{P} \mathbb{T}(\mathcal{F})$ by inclusion. The following lemma contains some basic results about $\left(\mathcal{F}^{<\omega}\right)^{+}$, which will be used implicitly.

Lemma 17 Let $\mathcal{F}$ be a filter on $\omega$ and $X \in\left(\mathcal{F}^{<\omega}\right)^{+}$.

1. If $F \in \mathcal{F}$, then $X \cap[F]^{<\omega} \in\left(\mathcal{F}^{<\omega}\right)^{+}$.

2. If $X=A \cup B$, then either $A \in\left(\mathcal{F}^{<\omega}\right)^{+}$or $B \in\left(\mathcal{F}^{<\omega}\right)^{+}$.

3. If $X=\left\{s_{n} \mid n \in \omega\right\}$ and $Y=\left\{y_{n} \mid n \in \omega\right\}$ is such that every $y_{n}$ is a non-empty subset of $s_{n}$. then $Y \in\left(\mathcal{F}^{<\omega}\right)^{+}$.

4. If $r_{\text {gen }}$ is an $(\mathbb{M}(\mathcal{F}), V)$-generic real, then $r_{\text {gen }}$ contains an element of $X$.

By point 1 above we get the following:

Lemma $18 \mathbb{P} \mathbb{T}(\mathcal{F})$ diagonalizes $\mathcal{F}$.

Given $A \subseteq[\omega]^{<\omega}$ we define minimal $(A) \subseteq A$ as the set of all minimal elements of $A$ with respect to the initial segment relation. Note that every element of $A$ contains an element of minimal $(A)$. We conclude that if $A \in$ $\left(\mathcal{F}^{<\omega}\right)^{+}$then minimal $(A) \in\left(\mathcal{F}^{<\omega}\right)^{+}$. 
Given $p \in \mathbb{P} \mathbb{T}(\mathcal{F}), s \in \operatorname{split}(p)$ and $D \subseteq \mathbb{P T}(\mathcal{F})$ an open dense subset, we define $E(D, p, s)=\operatorname{minimal}\left(\left\{t \backslash s \mid \exists q \leq p_{s}(\operatorname{stem}(q)=t \wedge q \in D)\right\}\right)$. We now have the following:

Lemma 19 If $p \in \mathbb{P} \mathbb{T}(\mathcal{F}), s \in \operatorname{split}(p)$ and $D \subseteq \mathbb{P T}(\mathcal{F})$ is an open dense subset, then $E(D, p, s) \in\left(\mathcal{F}^{<\omega}\right)^{+}$.

Proof. It would be enough to prove that $\left\{t \backslash s \mid \exists q \leq p_{s}(\operatorname{stem}(q)=t \wedge q \in D)\right\}$ is in $\left(\mathcal{F}^{<\omega}\right)^{+}$, which is immediate.

We can then prove the following:

Proposition 20 Let $\mathcal{F}$ be a filter.

1. Let $M$ be a countable elementary submodel, $p \in \mathbb{P} \mathbb{T}(\mathcal{F}), D \subseteq \mathbb{P} \mathbb{T}(\mathcal{F})$ an open dense subset with $p, D \in M$ and $s \in$ split $(p)$. There is $q \leq p$ with stem $(q)=s$ such that $q \Vdash$ " $\dot{G} \cap D \cap M \neq \emptyset "$.

2. $\mathbb{P T}(\mathcal{F})$ is proper.

Proof. Let $M$ be a countable elementary submodel, $p \in \mathbb{P} \mathbb{T}(\mathcal{F}), D \subseteq \mathbb{P T}(\mathcal{F})$ an open dense subset with $p, D \in M$ and $s \in \operatorname{split}(p)$. Since $p, D, s \in M$, it follows that $E(D, p, s) \in M$. For every $t$ such that $t \backslash s \in E(D, p, s)$, we choose $q(t) \in M \cap D$ such that $q(t) \leq p_{s}$ and the stem of $q(t)$ is $t$. Define $q=\bigcup\{q(t) \mid t \backslash s \in E(D, p, s)\}$. We will show that $q$ has the desired properties. Define $L=\{t \mid t \backslash s \in E(D, p, s)\}$, we will first prove that $q \in \mathbb{P} \mathbb{T}(\mathcal{F})$. Let $z \in q$ be a splitting node. If $z$ extends a $t \in L$, then $\operatorname{suc}_{q}(z)=\operatorname{suc}_{q(t)}(z)$, so $\operatorname{suc}_{q}(z) \in\left(\mathcal{F}^{<\omega}\right)^{+}$. Now, assume that $z$ does not extend an element of $L$, it is enough to prove that $\operatorname{suc}_{q}(z)=\operatorname{suc}_{p}(z)$. Let $n$ such that $z \in \operatorname{split}_{n}(q)$ and $w \in \operatorname{split}_{n+1}(q)$ such that $z \subseteq w$. Since $z$ does not extend an element of $L$, we know that $w$ can be extended to a $t \in L$, so $w \in q(t)$. Note that this argument shows that $\operatorname{stem}(q)=s$.

We will now prove that $q \Vdash$ " $\dot{G} \cap D \cap M \neq \emptyset$ ". Let $q_{1} \leq q$. We may assume that $q_{1} \in D$. Let $w=\operatorname{stem}\left(q_{1}\right)$. By construction, (recall that we took the minimal elements) we know there is $t \in L$ such that $t \subseteq w$, in this way $q_{1} \leq q(t)$, so $q_{1} \Vdash$ " $q(t) \in \dot{G} \cap D \cap M$ " and we are done.

The second part of the lemma follows by the first part and a fusion argument.

We will need the following lemma:

Lemma 21 Let $\mathcal{F}$ be a Canjar filter, $\left\{D_{n} \mid n \in \omega\right\}$ open dense subsets of $\mathbb{P} \mathbb{T}(\mathcal{F})$ and $p \in \mathbb{P} \mathbb{T}(\mathcal{F})$ with stem $(p)=s$. There are $q \in \mathbb{P} \mathbb{T}(\mathcal{F})$ and $\left\langle F_{n}\right\rangle_{n \in \omega}$ such that the following holds:

1. $q \leq p$ and $\operatorname{stem}(q)=\operatorname{stem}(p)$. 
2. $F_{n}$ is a finite subset of $[\omega]^{<\omega} \backslash\{\emptyset\}$ for every $n \in \omega$.

3. For every $n \in \omega$, if $t_{0} \in F_{n}$ and $t_{1} \in F_{n+1}$, then $\max \left(t_{0}\right)<\min \left(t_{1}\right)$.

4. $\operatorname{spsuc}_{q}(s)=\bigcup_{n \in \omega} F_{n}$.

5. If $t \in F_{n}$, then $q_{s \frown t} \in D_{n}$.

Proof. For every $n \in \omega$, define $X_{n}=E\left(D_{n}, p, s\right)$ (recall that $E(D, p, s)=$ minimal $\left.\left(\left\{t \backslash s \mid \exists q \leq p_{s}(\operatorname{stem}(q)=t \wedge q \in D)\right\}\right)\right)$. We know that $X_{n} \in\left(\mathcal{F}^{<\omega}\right)^{+}$. By the previous result, we can find an increasing function $f \in \omega^{\omega}$ such that if $Y_{n}=\left\{s \in X_{n} \mid s \subseteq[f(n-1), f(n))\right\}$ (for every $n \in \omega$ ) then $\bigcup_{n \in \omega} Y_{n} \in\left(\mathcal{F}^{<\omega}\right)^{+}$.

For every $t \in Y_{n}$, choose $q(t) \leq p_{s \frown t}$ such that $q(t) \in D_{n}$ and the stem of $q(t)$ is $s \cup t$. Define $q=\bigcup_{t \in Y} q(t)$.

By taking all the open dense sets to be the same, we obtain the following:

Corollary 22 Let $\mathcal{F}$ be a Canjar filter, $D$ an open dense subset of $\mathbb{P} \mathbb{T}(\mathcal{F})$, $p \in \mathbb{P} \mathbb{T}(\mathcal{F})$ with stem $(p)=s$. There is $q \in \mathbb{P} \mathbb{T}(\mathcal{F})$ such that the following holds:

1. $q \leq p$ and $\operatorname{stem}(q)=\operatorname{stem}(p)$.

2. If $t \in \operatorname{spsuc}_{q}(s)$ then $q_{s \frown t} \in D$.

We can now prove the following result:

Proposition 23 Let $\mathcal{B}$ be a $\mathfrak{b}$-family and $\mathcal{F}$ a $\mathcal{B}$-Canjar filter.

1. Let $p \in \mathbb{P} \mathbb{T}(\mathcal{F}), s \in$ split $_{m}(p)$ and $\dot{g}$ such that $p \Vdash " \dot{g} \in \omega^{\omega}$ ". There are $q \in \mathbb{P} \mathbb{T}(\mathcal{F}), f \in \mathcal{B}$ and $\left\langle F_{n}\right\rangle_{n \in \omega}$ such that the following holds:

(a) $q \leq p, s \in q$ and if $t \in p$ is incomparable with $s$, then $t \in q$ and $q_{t}=p_{t}$.

(b) For every $n \in \omega$, if $t_{0} \in F_{n}$ and $t_{1} \in F_{n+1}$, then $\max \left(t_{0}\right)<\min \left(t_{1}\right)$.

(c) $\operatorname{spsuc}_{q}(s)=\bigcup_{n \in \omega} F_{n}$.

(d) If $t \in F_{n}$ then $q_{t} \Vdash " \dot{g}(n)<f(n) "$.

2. $\mathbb{P} \mathbb{T}(\mathcal{F})$ preserves $\mathcal{B}$.

Proof. We first prove point 1 . Let $p \in \mathbb{P T}(\mathcal{F}), s \in \operatorname{split}_{m}(p)$ and $\dot{g}$ such that $p \Vdash " \dot{g} \in \omega^{\omega}$ ". Define $D_{n} \subseteq \mathbb{P} \mathbb{T}(\mathcal{F})$ as the set of all $q$ such that $q \Vdash$ " $\dot{g}(n)<\max (\operatorname{stem}(q)) "$. It is easy to see that each $D_{n}$ is an open dense subset of $\mathbb{P} \mathbb{T}(\mathcal{F})$. We now apply the previous lemma.

We will now prove that $\mathbb{P} \mathbb{T}(\mathcal{F})$ preserves $\mathcal{B}$ as an unbounded family. Let $p \in \mathbb{P} \mathbb{T}(\mathcal{F})$ and $\dot{g}$ such that $p \Vdash " \dot{g} \in \omega^{\omega}$ ". By the previous point and a fusion argument, we may assume that for every $s \in \operatorname{split}_{m}(p)$ there are $f_{s} \in \mathcal{B}$ and $\left\langle F_{n}^{s}\right\rangle_{n \in \omega}$ such that the following holds: 
1. Each $F_{n}^{s}$ is a finite subset of $p$.

2. $\operatorname{spsuc}_{p}(s)=\bigcup_{n \in \omega} F_{n}^{s}$.

3. if $t \in F_{n}^{s}$ then $p_{t} \Vdash " \dot{g}(n)<f_{s}(n) "$.

Since $\mathcal{B}$ is a $\mathfrak{b}$-family, we can find $f \in \mathcal{B}$ such that $f_{s} \leq^{*} f$ for every $s \in$ $\operatorname{split}(p)$. For every $s \in \operatorname{split}(p)$, let $m_{s}$ such that if $i>m_{s}$ then $f_{s}(i) \leq f(i)$. We can recursively build $q \leq p$ such that $\operatorname{split}(q)=\operatorname{split}(p) \cap q$ and $\operatorname{suc}_{q}(s)=$ $\bigcup_{n>m_{s}} F_{n}^{s}$. It is easy to see that $q$ forces that $\dot{g}$ does not dominate $f$.

It is worth mentioning that $\mathbb{P T}(\mathcal{F})$ may add dominating reals for certain filters $\mathcal{F}$. The simplest example is taking $\mathcal{F}$ to be $(\text { fin } \times \text { fin })^{*}$.

The following is a very useful fact about our forcings:

Lemma 24 (Pure decision property) Let $\mathcal{F}$ be a Canjar filter, $p \in \mathbb{P} \mathbb{T}(\mathcal{F})$ and $A$ a finite set. If $\dot{x}$ is a $\mathbb{P T}(\mathcal{F})$-name such that $p \Vdash$ " $\dot{x} \in A$ " then there are $q \leq p$ and $a \in A$ such that stem $(q)=\operatorname{stem}(p)$ and $q \Vdash " \dot{x}=a "$.

Proof. Let $D$ be the set of all $q \in \mathbb{P} \mathbb{T}(\mathcal{F})$ for which there is $a_{q} \in A$ such that $q \Vdash$ " $\dot{x}=a_{q} "$. Since $D$ is an open dense set, by the previous results, we can find $\bar{p} \leq p$ with the following properties:

1. $\operatorname{stem}(\bar{p})=\operatorname{stem}(p)$.

2. If $t \in \operatorname{split}_{1}(\bar{p})$ then $\bar{p}_{t} \in D$.

Finally, since $A$ is finite, we can find $q \leq p$ with stem $(q)=\operatorname{stem}(p)$ and $a \in A$ such that $q_{t} \Vdash$ " $\dot{x}=a$ " for every $t \in \operatorname{split}_{1}(q)$. It follows that $q \Vdash " \dot{x}=a$ ".

By $\mathcal{K}_{\sigma}$ we denote the ideal generated by all $\sigma$-compact sets on the Baire space. For every function $L: \omega^{<\omega} \longrightarrow \omega$, let $K(L)$ be the set defined as $\left\{x \in \omega^{\omega} \mid \forall^{\infty} n(x(n) \leq L(x \uparrow n))\right\}$. It is easy to see that $K(L) \in \mathcal{K}_{\sigma}$. It is well known that for every $K \in \mathcal{K}_{\sigma}$ there is $f \in \omega^{\omega}$ such that $\mathcal{K} \subseteq\left\{g \in \omega^{\omega} \mid g \leq^{*} f\right\}$ (see [3] page 6). The following well known result follows from this remark:

Lemma 25 Let $\mathcal{B} \subseteq \omega^{\omega}$ be an unbounded family. If $\mathbb{P}$ is a forcing that preserves $\mathcal{B}$, then $\mathbb{P} \Vdash " \mathcal{B} \notin \mathcal{K}_{\sigma} "$.

If $T$ is a finite tree, we will denote the set of its maximal nodes as $[T]$. We will need the following notion:

Definition 26 Let $p \in \mathbb{P} \mathbb{T}(\mathcal{F})$ and $T \subseteq p$ a finite tree such that $[T] \subseteq$ split $(p)$. We say that $q \leq_{T} p$ if the following conditions hold: 
1. $q \leq p$.

2. $T \subseteq q$.

3. $T \cap \operatorname{split}(q)=T \cap \operatorname{split}(p)$.

We fix a canonical bijection $d: \omega \longrightarrow \omega^{<\omega}$ such that if $d(m) \subseteq d(n)$ then $m \leq n$. Given $p \in \mathbb{P T}(\mathcal{F})$ and $n \in \omega$, we define the set $\widetilde{T}(p, n)=$ $\left\{s \in \operatorname{split}(p) \mid d^{-1}(s) \leq n\right\}$. Let $T(p, n) \subseteq \omega^{<\omega}$ be the smallest tree such that $\widetilde{T}(p, n) \subseteq T(p, n)$. It is clear that $T(p, n)$ is a finite subtree of $p$ such that $[T(p, n)] \subseteq \operatorname{split}(p)$. It is easy to see that if $q \leq_{T(p, n)} p$ and $n \leq m$, then $T(p, n) \subseteq T(q, m)$.

Let $p \in \mathbb{P T}(\mathcal{F})$ and $\mathcal{B}$ a $\mathfrak{b}$-family. We define the game $\mathcal{G}(\mathcal{F}, p, \mathcal{B})$ as follows:

\begin{tabular}{|l|l|l|l|l|l|l|l|}
\hline $\mathrm{I}$ & $p_{0}$ & & $p_{1}$ & & $p_{2}$ & & $\ldots$ \\
\hline $\mathrm{II}$ & & $n_{0}$ & & $n_{1}$ & & $n_{2}$ & \\
\hline
\end{tabular}

1. $p_{i} \in \mathbb{P} \mathbb{T}(\mathcal{F})$ and $n_{i} \in \omega$ for every $i \in \omega$.

2. $p_{0}=p$.

3. $\left\langle n_{i}\right\rangle_{i \in \omega}$ is increasing.

4. $p_{m+1} \leq_{T_{m}} p_{m}$ where $T_{m}=T\left(p_{m}, n_{m}\right)$.

The player II will win the game $\mathcal{G}(\mathcal{F}, p, \mathcal{B})$ if $\bigcup T_{m} \in \mathbb{P T}(\mathcal{F})$ and $f \in \mathcal{B}$ where $f$ is the function given by $f(i)=n_{i}$.

Proposition 27 Let $\mathcal{F}$ be a filter, $p \in \mathbb{P} \mathbb{T}(\mathcal{F})$ and $\mathcal{B}$ a $\mathfrak{b}$-family. If $\mathcal{F}$ is $\mathcal{B}$ Canjar, then I does not have a winning strategy in $\mathcal{G}(\mathcal{F}, p, \mathcal{B})$.

Proof. Let $\sigma$ be a strategy for player I, we must prove that player II can defeat $\sigma$. Define $\left\{p(s) \mid s \in \omega^{<\omega}\right\} \subseteq \mathbb{P} \mathbb{T}(\mathcal{F})$ as follows:

1. $p(\emptyset)=p$.

2. If $s=\left\langle n_{0}, \ldots, n_{m}\right\rangle$ then $p(s)$ is the tree played by player I at the $m$-step if he is playing according to $\sigma$ and II plays $n_{i}$ at the step $i$ for $i \leq m$.

Let $r_{\text {gen }}$ be an $(\mathbb{M}(\mathcal{F}), V)$-generic real (note that $r_{\text {gen }}$ is generic for $\mathbb{M}(\mathcal{F})$, not for $\mathbb{P T}(\mathcal{F})$ ). In $V\left[r_{\text {gen }}\right]$ we define a function $L: \omega^{<\omega} \longrightarrow \omega$ as follows: Let $s=\left\langle n_{i}\right\rangle_{i<m} \in \omega^{<\omega}$, we look at $p(s)$, assume that player II plays $n_{i}$ at the step $i$ for $i<m$ and player $\mathbf{I}$ is following $\sigma$. Let $T_{m}$ be the tree defined 
so far (as in the definition in the game). Let $t \in T_{m} \cap \operatorname{split}(p(s))$ and since $\operatorname{spsuc}_{p(s)}(t) \in\left(\mathcal{F}^{<\omega}\right)^{+}$, it follows by genericity that there is $m_{s}(t) \in \omega$ such that there is $u_{s}(t) \in \operatorname{spsuc}_{p(s)}(t)$ for which $u_{s}(t) \subseteq\left(r_{g e n} \backslash m\right) \cap m_{s}(t)$. Let $L(s)$ such that $d^{-1}\left(t \cup u_{s}(t)\right)<L(s)$ for all $t \in T_{m} \cap \operatorname{split}(p(s))$.

Since $\mathcal{F}$ is $\mathcal{B}$-Canjar, we can find $f \in \mathcal{B}$ and $(z, F) \in \mathbb{M}(\mathcal{F})$ such that $(z, F) \Vdash$ " $f \notin K(\dot{L})$ ". We claim that if II plays $f(n)$ at the $n$-step of the game, then she will win the match. Let $q=\bigcup T_{m}$, we must show that $q \in \mathbb{P T}(\mathcal{F})$.

Let $t \in q$ be a splitting node, find $n \in \omega$ such that $t \in T_{n}$ (where $T_{n}$ is defined as in the game). We must prove that $\operatorname{spsuc}_{q}(t) \in\left(\mathcal{F}^{<\omega}\right)^{+}$. Let $H \in \mathcal{F}$ and note that $(z, F \cap H) \Vdash$ " $f \notin K(\dot{L})$ ". We know we can find $\left(z \cup z_{0}, G\right) \in \mathbb{M}(\mathcal{F})$ and $m \in \omega$ such that the following conditions hold:

1. $m>n, \max (z)$.

2. $\max (z)<\min \left(z_{0}\right)$.

3. $\left(z \cup z_{0}, G\right) \leq(z, F \cap H)$ (in particular, $\left.z_{0} \subseteq H\right)$.

4. $\left(z \cup z_{0}, G\right) \Vdash " \dot{L}(f\lceil m)<f(m) "$.

It follows by all the definitions that $T_{m+1}$ will contain an element of the set $\operatorname{spsuc}_{p(f\lceil m)}(t)$. Note that such element must be a subset of $z_{0}$, so in particular is a subset of $H$. This finishes the proof.

The previous argument was motivated by the fact that Canjar is equivalent to its "game version". This interesting result is a corollary of the theorems proved by Chodounský, Repovš and Zdomskyy in [19], we will comment more about this in the next section.

Definition 28 We say that $D \subseteq \mathbb{P} \mathbb{T}(\mathcal{F})$ is purely dense if the following conditions hold:

1. If $p \in D$ and $q \leq p$ then $q \in D$ ( $D$ is open).

2. For every $p \in \mathbb{P T}(\mathcal{F})$ and for every finite tree $T \subseteq p$ such that $[T] \subseteq$ split $(p)$, there is $q \leq_{T} p$ such that $q \in D$.

Intuitively, the purely dense sets are the open sets we can get in by only using the pure decision property.

We will now prove that if $\mathcal{F}$ is a Canjar filter, then $\mathbb{P} \mathbb{T}(\mathcal{F})$ does not add Cohen or random reals. Recall the following notion:

Definition 29 We say that $c \in \omega^{\omega}$ is a half-Cohen real over $V$ if for every $f \in \omega^{\omega} \cap V$ the set $\{n \mid c(n)=f(n)\}$ is infinite. 
Obviously every Cohen real over $V$ is half-Cohen over $V$. It can be proved that if one adds an unbounded real and then a half-Cohen, a Cohen real is added (see [5]). It was a long standing question of Fremlin if it was possible to add a half-Cohen real without adding a Cohen real. This problem was finally solved positively by Zapletal in 63 . We will prove that if $\mathcal{F}$ is Canjar, then $\mathbb{P T}(\mathcal{F})$ does not add a half-Cohen real. We start with the following lemma:

Lemma 30 Let $\mathcal{F}$ be a Canjar filter and $\dot{a}$ a $\mathbb{P} T(\mathcal{F})$-name for a natural number. The set $D=\{p \in \mathbb{P} \mathbb{T}(\mathcal{F}) \mid \exists k \in \omega(p \Vdash " \dot{a} \neq k ")\}$ is purely dense.

Proof. Let $p \in \mathbb{P} \mathbb{T}(\mathcal{F})$ and $T \subseteq p$ a finite tree such that every maximal node of $T$ is an element of split $(p)$. We need to find $q \leq_{T} p$ such that $q \in D$. Let $n=|T \cap \operatorname{split}(p)|$ and for every $s \in T \cap \operatorname{split}(p)$ let $p(s) \leq p_{s}$ be the biggest subtree of $p_{s}$ such that every $t \in T \cap p(s)$ is a restriction of $s$. Using the pure decision property, for every $s \in T \cap \operatorname{split}(p)$ we can find $q(s) \in \mathbb{P T}(\mathcal{F})$ and $k_{s} \in \omega$ with the following properties:

1. $q(s) \leq p(s)$ and the stem of $q(s)$ is $s$.

2. $q(s) \Vdash " \dot{a} \leq n+1 "$ or $q(s) \Vdash “ \dot{a}>n+1 "$.

3. If $q(s) \Vdash " \dot{a} \leq n+1 "$ then $q(s) \Vdash " \dot{a}=k_{s} "$.

Since $n=|T \cap \operatorname{split}(p)|$, we can find $k \leq n+1$ such that $k \neq k_{s}$ for every $s \in T \cap \operatorname{split}(p)$. Let $q=\bigcup\{q(s) \mid s \in T \cap \operatorname{split}(p)\}$. Note that $q \leq_{T} p$ and $q \Vdash$ " $\dot{a} \neq k "$, this finishes the proof.

We can now prove the following:

Proposition 31 If $\mathcal{F}$ is a Canjar filter, then $\mathbb{P} \mathbb{T}(\mathcal{F})$ does not add half-Cohen reals.

Proof. Let $p \in \mathbb{P} \mathbb{T}(\mathcal{F})$ and $\dot{f}$ a $\mathbb{P} \mathbb{T}(\mathcal{F})$-name for an element of $\omega^{\omega}$. We must prove that there is $q \leq p$ and $g \in \omega^{\omega}$ such that $q \Vdash$ " $\dot{f} \cap g=\emptyset "$ ". For every $n \in \omega$, define $D_{n}=\{q \in \mathbb{P} \mathbb{T}(\mathcal{F}) \mid \exists k \in \omega(q \Vdash$ " $\dot{f}(n) \neq k ")\}$, note that each $D_{n}$ is purely dense by the previous lemma.

Let $\preceq$ be any well order of $\mathbb{P} \mathbb{T}(\mathcal{F})$. We will recursively define a strategy $\sigma$ for player I on the game $\mathcal{G}\left(\mathcal{F}, p, \omega^{\omega}\right)$ as follows:

1. I starts by playing $p$.

2. Assume we are at round $m+1$ and I has played $p_{0}, \ldots, p_{m}$ while player II has played $n_{0}, \ldots, n_{m-1}$. If player II plays $n_{m}$, then player I plays $p_{m+1}$ where $p_{m+1}$ is the $\preceq$-least element of $\mathbb{P T}(\mathcal{F})$ such that $p_{m+1} \in D_{m}$ and $p_{m+1} \leq_{T_{m}} p_{m}\left(\right.$ where $\left.T_{m}=T\left(p_{m}, n_{m}\right)\right)$. 
Since $\mathcal{F}$ is Canjar, we know that $\sigma$ is not a winning strategy for player I, which means that there is a function $d \in \omega^{\omega}$ such that if player II plays $d(n)$ at the $n$-step then she will win. Let $q$ be the condition constructed at the end of the game. Note that $q \in \bigcap_{\in \omega} D_{n}$ in this way, we can define a function $g: \omega \longrightarrow \omega$ such that $q \Vdash " \dot{f}(n) \neq g(n)$ ” for every $n \in \omega$. This finishes the proof.

We will now prove that if $\mathcal{F}$ is Canjar, then $\mathbb{P T}(\mathcal{F})$ does not add bounded eventually different reals. By $F n(\omega)$ we will denote the set of all functions $z: a \longrightarrow \omega$ such that $a \in[\omega]^{<\omega}$. We will need the following lemma:

Lemma 32 Let $m \in \omega, g \in \omega^{\omega}$ and $\dot{f}$ be a $\mathbb{P} \mathbb{T}(\mathcal{F})$-name for a function bounded by g. The set $D=\{p \in \mathbb{P} \mathbb{T}(\mathcal{F}) \mid \exists z \in F n(\omega)(m \cap \operatorname{dom}(z)=\emptyset \wedge p \Vdash " \dot{f} \cap z \neq$ $\emptyset ")\}$ is purely dense.

Proof. Let $p \in \mathbb{P} \mathbb{T}(\mathcal{F})$ and $T \subseteq p$ a finite tree such that every maximal node of $T$ is an element of split $(p)$. We need to find $q \leq_{T} p$ such that $q \in D$. Let $n=|T \cap \operatorname{split}(p)|$ and for every $s \in T \cap \operatorname{split}(p)$ let $p(s) \leq p_{s}$ be the biggest subtree of $p_{s}$ such that every $t \in T \cap p(s)$ is a restriction of $s$. Let $T \cap \operatorname{split}(p)=$ $\left\{s_{i} \mid i<n\right\}$. Using the pure decision property, for every $s_{i} \in T \cap \operatorname{split}(p)$ we can find $q\left(s_{i}\right) \in \mathbb{P} \mathbb{T}(\mathcal{F})$ and $k_{i} \in \omega$ with the following properties:

1. $q\left(s_{i}\right) \leq p\left(s_{i}\right)$ and the stem of $q\left(s_{i}\right)$ is $s_{i}$.

2. $q\left(s_{i}\right) \Vdash " \dot{f}(m+i)=k_{i} "$.

Note that $q\left(s_{i}\right)$ can be found (with the pure decision property) since there are only finitely many possibilities for $\dot{f}(m+i)$. We now define a function $z$ : $[m, m+n) \longrightarrow \omega$ given by $z(m+i)=k_{i}$. Let $q=\bigcup\{q(s) \mid s \in T \cap$ split $(p)\}$. Note that $q \leq_{T} p$ and $q \Vdash " \dot{f} \cap z \neq \emptyset "$, this finishes the proof.

We now can prove the following:

Proposition 33 If $\mathcal{F}$ is a Canjar filter, then $\mathbb{P} \mathbb{T}(\mathcal{F})$ does not add bounded eventually different reals.

Proof. Let $p \in \mathbb{P} \mathbb{T}(\mathcal{F}), g \in \omega^{\omega}$ and $\dot{f}$ an $\mathbb{P} \mathbb{T}(\mathcal{F})$-name for an element of $\omega^{\omega}$ bounded by $g$. We must prove that there is $q \leq p$ and $h \in \omega^{\omega}$ such that $q \Vdash "|\dot{f} \cap h|=\omega "$. For every $m \in \omega$, define $D_{m}=\{p \in \mathbb{P} \mathbb{T}(\mathcal{F}) \mid \exists z \in$ $F n(\omega)(m \cap \operatorname{dom}(z)=\emptyset \wedge p \Vdash " \dot{f} \cap z \neq \emptyset ")\}$, which we already know is a purely dense set.

Let $\preceq$ be any well order of $\mathbb{P} \mathbb{T}(\mathcal{F})$. We will recursively define a strategy $\sigma$ for player I on the game $\mathcal{G}\left(\mathcal{F}, p, \omega^{\omega}\right)$ as follows:

1. I starts by playing $p$. 
2. Assume we are at round $m+1$ and $I$ has played $p_{0}, \ldots, p_{m}$ while player II has played $n_{0}, \ldots, n_{m-1}$. If player II plays $n_{m}$, then player I plays $p_{m+1}$ where $p_{m+1}$ is the $\preceq$-least element of $\mathbb{P} \mathbb{T}(\mathcal{F})$ such that $p_{m+1} \in D_{m}$ and $p_{m+1} \leq_{T_{m}} p_{m}\left(\right.$ where $\left.T_{m}=T\left(p_{m}, n_{m}\right)\right)$.

Since $\mathcal{F}$ is Canjar, we know that $\sigma$ is not a winning strategy for player I, hence there is a function $d \in \omega^{\omega}$ such that if player II plays $d(n)$ at the $n$-step then, she will win. Let $q$ be the condition constructed at the end of the game. Note that $q \in \bigcap_{\in \omega} D_{n}$, this means that for every $n \in \omega$, we can find a finite function $z$ such that $\operatorname{dom}(z)=\emptyset$ and $q \Vdash " z \cap \dot{f} \neq \emptyset "$. We can now easily find a function $h: \omega \longrightarrow \omega$ such that $q \Vdash "|\dot{f} \cap h|=\omega "$.

It is well known that adding a random real adds a bounded eventually different real. In this way we conclude the following:

Corollary 34 If $\mathcal{F}$ is a Canjar filter, then $\mathbb{P T}(\mathcal{F})$ does not add random reals.

We also have the following lemma:

Lemma 35 Let $T \subseteq \omega^{<\omega}$ be a finite tree, $p \in \mathbb{P T}(\mathcal{F})$ such that $[T] \subseteq$ split $(p)$, let $n=\mid$ split $(p) \cap T \mid$ and let $A$ be a finite set. If $\dot{x}$ is a $\mathbb{P T}(\mathcal{F})$-name such that $p \Vdash$ " $\dot{x} \in A$ ", there is $q \leq_{T} p$ and $B \in[A]^{n}$ such that $q \Vdash$ " $\dot{x} \in B$ ".

Proof. The lemma easily follows by applying the pure decision property $n$-many times.

\section{Preservation of $P$-points}

Let $\mathcal{U}$ be an ultrafilter and $\mathbb{P}$ a partial order. We say that $\mathbb{P}$ preserves $\mathcal{U}$ if $\mathcal{U}$ is the base of an ultrafilter after forcing with $\mathbb{P}$. It is well known that no ultrafilter is preserved by Cohen, random, Silver or forcings adding a dominating real. Also there is an ultrafilter that is destroyed by any forcing that adds a new real (see [3]). On the other hand, certain forcings may preserve some ultrafilters, this is the case for Sacks and Miller forcings. The preservation of $P$-points is particularly interesting in light of the following theorem of Shelah (see 58] or [3]):

Proposition 36 (Shelah) Let $\delta$ be a limit ordinal, $\left\langle\mathbb{P}_{\alpha}, \dot{\mathbb{Q}}_{\alpha} \mid \alpha<\delta\right\rangle$ a countable support iteration of proper forcings and let $\mathcal{U}$ be a P-point. If $\mathbb{P}_{\alpha} \Vdash " \dot{\mathbb{Q}}_{\alpha}$ preserves $\mathcal{U}$ " for every $\alpha<\delta$, then $\mathbb{P}_{\delta}$ preserves $\mathcal{U}$.

In particular, forcings that preserve $P$-points do not add Cohen or random reals, even in the iteration. It is well known that Sacks forcing preserves $P$ points and Miller forcing preserves an ultrafilter $\mathcal{U}$ if and only if $\mathcal{U}$ is a $P$-point 
(see [50]). Note that if $\mathbb{P}$ diagonalizes $\mathcal{U}$, then $\mathbb{P}$ does not preserve $\mathcal{U}$, however, it is possible to not preserve an ultrafilter without diagonalizing it. For more on preservation of ultrafilters, the reader may consult [43], [50], [3], [48, 62] and 64 .

In light of the results of the previous section, it might be tempting to conjecture that if $\mathcal{F}$ is a Canjar filter, then $\mathbb{P T}(\mathcal{F})$ preserves $P$-points. However, this is not the case: the simplest example is to take $\mathcal{U}$ a Canjar $P$-point, since $\mathbb{P} \mathbb{T}(\mathcal{U})$ diagonalizes $\mathcal{U}$, it follows that $\mathbb{P T}(\mathcal{U})$ does not preserve $\mathcal{U}$. In this section, we will find a condition on a filter $\mathcal{F}$ that guarantees preserving a certain $P$-point.

We will need the following result, which is a particular case of lemma 11.

Lemma 37 Let $\mathcal{F}$ be a Canjar filter and $X \in\left(\mathcal{F}^{<\omega}\right)^{+}$. There is $Y \subseteq X$ such that $Y \in\left(\mathcal{F}^{<\omega}\right)^{+}$and for every $n \in \omega$, the set $\{s \in Y \mid s \cap n \neq \emptyset\}$ is finite.

We can now prove the following:

Lemma 38 Let $\mathcal{F}$ be a Canjar filter, $p \in \mathbb{P T}(\mathcal{F})$ and $c:$ split $(p) \longrightarrow 2$. There is $q \leq p$ such that split $(q)$ is c-monochromatic.

Proof. Assume there is no $q \leq p$ such that $\operatorname{split}(q)$ is 0 -monochromatic. We will prove that there is $q \leq p$ such that $\operatorname{split}(q)$ is 1 -monochromatic. Given $s \in \operatorname{split}(p)$, we define $X(s)$ as the set of all $t \backslash s$ for which $t \in \operatorname{split}(p)$, $s \subseteq t$ and $c(t)=1$. We claim that $X(s) \in\left(\mathcal{F}^{<\omega}\right)^{+}$. Assume this is not the case, so there is $A \in \mathcal{F}$ such that $A$ does not contain any element of $X(s)$. Let $q \leq p_{s}$ such that if $t \in \operatorname{split}(q)$, then $t \backslash s \subseteq A$. It follows that if $t \in \operatorname{split}(q)$, then $c(t)=0$, so split $(q)$ is 0 -monochromatic, which is a contradiction. We conclude that $X(s) \in\left(\mathcal{F}^{<\omega}\right)^{+}$for every $s \in$ split $(p)$. By the previous result, we can find $Y(s) \subseteq X(s)$ such that $Y(s) \in\left(\mathcal{F}^{<\omega}\right)^{+}$and for every $n \in \omega$, the set $\{z \in Y \mid z \cap n \neq \emptyset\}$ is finite. The proof follows by a simple fusion argument.

Let $\mathcal{F}$ be a filter. The Canjar game $\mathcal{G}_{\text {Canjar }}(\mathcal{F})$ is defined as follows:

\begin{tabular}{|l|l|l|l|l|l|l|l|}
\hline $\mathrm{I}$ & $X_{0}$ & & $X_{1}$ & & $X_{2}$ & & $\ldots$ \\
\hline $\mathrm{II}$ & & $Y_{0}$ & & $Y_{1}$ & & $Y_{2}$ & \\
\hline
\end{tabular}

Where $X_{i} \in\left(\mathcal{F}^{<\omega}\right)^{+}$and $Y_{i} \in\left[X_{i}\right]^{<\omega}$ for every $i \in \omega$. The player II wins the game $\mathcal{G}_{\text {Canjar }}(\mathcal{F})$ if $\bigcup_{n \in \omega} Y_{n} \in\left(\mathcal{F}^{<\omega}\right)^{+}$. In [19], Chodounský, Repovš and Zdomskyy showed that sets in $\left(\mathcal{F}^{<\omega}\right)^{+}$naturally correspond to open covers of $\mathcal{F}$ (viewed as a subspace of $\wp(\omega)$ ). Moreover, they proved that a filter $\mathcal{F}$ is Canjar if and only if $\mathcal{F}$ has the Menger property (see [56] for the definition of Menger property). In this way, the Canjar game is just a particular case of the Menger game that has been extensively studied in topology. 
Proposition $39([\mathbf{1 9}])$ Let $\mathcal{F}$ be a filter. The following are equivalent:

1. $\mathcal{F}$ is Canjar.

2. $\mathcal{F}$ is Menger.

3. Player I does not have a winning strategy in $\mathcal{G}_{\text {Canjar }}(\mathcal{F})$.

We will now prove the following lemma:

Lemma 40 Let $\mathcal{F}$ be a Canjar filter, $p \in \mathbb{P} \mathbb{T}(\mathcal{F}), \dot{B}$ a $\mathbb{P} \mathbb{T}(\mathcal{F})$-name such that $p \Vdash " \dot{B} \in[\omega]^{\omega} "$ and $s \in$ split $(p)$. There are $q \in \mathbb{P} \mathbb{T}(\mathcal{F}), B_{s} \subseteq \omega$ and $\left\langle F_{n}\right\rangle_{n \in \omega}$ such that the following holds:

1. $q \leq p$ and $\operatorname{stem}(q)=s$.

2. $F_{n}$ is a finite subset of $[\omega]^{<\omega} \backslash\{\emptyset\}$ for every $n \in \omega$.

3. For every $n \in \omega$, if $t_{0} \in F_{n}$ and $t_{1} \in F_{n+1}$, then $\max \left(t_{0}\right)<\min \left(t_{1}\right)$.

4. $\operatorname{spsuc}_{q}(s)=\bigcup_{n \in \omega} F_{n}$.

5. If $t \in F_{n}$, then $q_{s-t} \Vdash " \dot{B} \cap(n+1)=B_{s} \cap(n+1)$ ".

Proof. We define $\sigma$ a strategy for player I in $\mathcal{G}_{\text {Canjar }}(\mathcal{F})$ as follows:

1. Using the pure decision property, player I finds $q_{0} \leq p$ with stem $(q)=s$ and $w_{0}$ such that $q^{0} \Vdash$ " $\dot{B} \cap 1=w_{0}$ " and plays $X_{0}=\operatorname{spsuc}_{q^{0}}(s)$.

2. Assume that player II plays $Y_{0} \in\left[X_{0}\right]^{<\omega}$. Let $l_{0} \in \omega$ be the least such that $\bigcup Y_{0} \subseteq l_{0}$ and $Z_{0}=\left\{t \in X_{0} \mid t \cap l_{0}=\emptyset\right\} \in\left(\mathcal{F}^{<\omega}\right)^{+}$. Let $\bar{q}^{1} \leq q^{0}$ be the condition defined as $\bar{q}^{1}=\bigcup_{t \in Z_{0}}\left(q^{0}\right)_{s \frown t}$. Using the pure decision property, player I finds $q^{1} \leq \bar{q}^{1}$ with stem $(q)=s$ and $w_{1}$ such that $q^{1} \Vdash " \dot{B} \cap 2=w_{1}$ " and plays $X_{1}=$ spsuc $_{q^{1}}(s)$.

3. In general, at step $n$, the player I has constructed a decreasing sequence $\left\langle q^{i}\right\rangle_{i \leq n}$ where stem $\left(q^{n}\right)=s$, an increasing sequence $\left\langle w_{i}\right\rangle_{i \leq n}$ such that $q^{n} \Vdash$ " $\dot{B} \cap(n+1)=w_{n}$ " has played the sequence $\left\langle X_{i}\right\rangle_{i \leq n}$ where $X_{i}=$ $\operatorname{spsuc}_{q^{i}}(s)$, he has also constructed an increasing sequence $\left\langle l_{i}\right\rangle_{i \leq n}$ such that $Y_{i} \subseteq\left[l_{i-1}, l_{i}\right)$ (where $l_{-1}=0$ and $Y_{i}$ is the response of player II at round $i$ ). Assume that player II plays $Y_{n} \in\left[X_{n}\right]^{<\omega}$. Let $l_{n} \in \omega$ be the least such that $\bigcup Y_{n} \subseteq l_{n}$ and $Z_{n}=\left\{t \in X_{n} \mid t \cap l_{n}=\emptyset\right\} \in\left(\mathcal{F}^{<\omega}\right)^{+}$. Let $\bar{q}^{n+1} \leq q^{n}$ be the condition defined as $\bar{q}^{n+1}=\bigcup_{t \in Z_{n}}\left(q^{n}\right)_{s \frown t}$. Using the pure decision property, player I finds $q^{n+1} \leq \bar{q}^{n}$ with stem $(q)=s$ and $w_{n+1}$ such that $q^{n+1} \Vdash$ " $\dot{B} \cap(n+2)=w_{n+1}$ " and plays $X_{n+1}=\operatorname{spsuc}_{q^{n+1}}(s)$. 
Since $\mathcal{F}$ is Canjar, we know that $\sigma$ is not a winning strategy for the Canjar game. Consider a run in which player II defeated the strategy. Let $q=$ $\bigcup_{s \in Y_{i}}\left(q^{i}\right)_{s \frown t}$ and note that $q \in \mathbb{P T}(\mathcal{F})$ since player II won the game. Define $F_{i}=Y_{i}$ and $B_{s}=\bigcup_{i \in \omega} w_{i}$, it is clear that this are the items we were looking for.

Let $\mathcal{U}$ be an ultrafilter. Recall that the $P$-point game $\mathcal{G}_{\text {P-point }}(\mathcal{U})$ is defined as follows:

\begin{tabular}{|l|l|l|l|l|l|}
\hline $\mathrm{I}$ & $W_{0}$ & & $W_{1}$ & & $\ldots$ \\
\hline $\mathrm{II}$ & & $z_{0}$ & & $z_{1}$ & \\
\hline
\end{tabular}

Where $W_{i} \in \mathcal{U}$ and $z_{i} \in\left[W_{i}\right]^{<\omega}$. The player II will win the game $\mathcal{G}_{P \text {-point }}(\mathcal{U})$ if $\bigcup_{m \in \omega} z_{m} \in \mathcal{U}$. It is well known that player II can not have a winning strategy for this game. The following is a well known result of Galvin and Shelah (see [3] for a proof):

Proposition 41 (Galvin, Shelah) Let $\mathcal{U}$ be an ultrafilter. The following are equivalent:

1. $\mathcal{U}$ is a P-point.

2. Player I does not have a winning strategy in $\mathcal{G}_{P \text {-point }}(\mathcal{U})$.

Let $\mathcal{U}$ be an ultrafilter and $\mathcal{F}$ a filter. We will now define the game $\mathcal{H}(\mathcal{U}, \mathcal{F})$, which is a fusion between the $P$-point game and the game for $\mathbb{P} \mathbb{T}(\mathcal{F})$. The game is defined as follows:

\begin{tabular}{|l|l|l|l|l|l|l|l|l|l|}
\hline $\mathrm{I}$ & $W_{0}$ & & $p_{0}$ & & $W_{1}$ & & $p_{1}$ & & $\ldots$ \\
\hline $\mathrm{II}$ & & $z_{0}$ & & $n_{0}$ & & $z_{1}$ & & $n_{1}$ & \\
\hline
\end{tabular}

Where the following conditions hold for every $i \in \omega$ :

1. $W_{i} \in \mathcal{U}$.

2. $z_{i} \in\left[W_{i}\right]^{<\omega}$.

3. $p_{i} \in \mathbb{P} \mathbb{T}(\mathcal{F})$.

4. $\left\langle n_{i}\right\rangle_{i \in \omega}$ is an increasing sequence of natural numbers.

5. $p_{m+1} \leq_{T_{m}} p_{m}$ where $T_{m}=T\left(p_{m}, n_{m}\right)$. 
The player II will win the game $\mathcal{H}(\mathcal{U}, \mathcal{F})$ if $\bigcup_{m \in \omega} T_{m} \in \mathbb{P} \mathbb{T}(\mathcal{F})$ and $\bigcup_{m \in \omega} z_{m} \in \mathcal{U}$.

Definition 42 Let $\mathcal{F}$ be a filter and $\mathcal{U}$ an ultrafilter. We will say that $\mathcal{F}$ is an $\mathcal{U}$-Canjar filter if player I has no winning strategy in $\mathcal{H}(\mathcal{U}, \mathcal{F})$.

Note that the previous notion is only of interest when $\mathcal{F}$ is Canjar and $\mathcal{U}$ is a $P$-point. It is easy to see that if $\mathcal{U}$ is an ultrafilter, then $\mathcal{U}$ is not $\mathcal{U}$-Canjar (and will also follow by the next result). Our interest in $\mathcal{U}$-Canjar filters is that (as we are about to prove), its Miller forcing preserves $\mathcal{U}$. Our proof is based on the argument of Miller that the superperfect forcing preserves $P$-points (see [50]).

Proposition 43 If $\mathcal{U}$ is a P-point and $\mathcal{F}$ is an $\mathcal{U}$-Canjar filter, then $\mathbb{P} \mathbb{T}(\mathcal{F})$ preserves $\mathcal{U}$.

Proof. Let $p \in \mathbb{P} \mathbb{T}(\mathcal{F})$ and $\dot{B}$ a $\mathbb{P} \mathbb{T}(\mathcal{F})$-name such that $p \Vdash " \dot{B} \in[\omega]^{\omega}$ ". By lemma 40, we may assume that for every $s \in \operatorname{split}(p)$, there is $B_{s} \subseteq \omega$ and $\left\langle F_{n}^{s}\right\rangle_{n \in \omega}$ with the following properties:

1. $F_{n}^{s}$ is a finite subset of $[\omega]^{<\omega} \backslash\{\emptyset\}$ for every $n \in \omega$.

2. For every $n \in \omega$, if $t_{0} \in F_{n}^{s}$ and $t_{1} \in F_{n+1}^{s}$, then $\max \left(t_{0}\right)<\min \left(t_{1}\right)$.

3. $\operatorname{spsuc}_{p}(s)=\bigcup_{n \in \omega} F_{n}^{s}$.

4. If $t \in F_{n}^{s}$, then $p_{s \frown t} \Vdash " \dot{B} \cap(n+1)=B_{s} \cap(n+1)$ ".

Furthermore, by the lemma 38 we may assume that either $B_{s} \in \mathcal{U}$ for all $s \in \operatorname{split}(p)$ or $B_{s} \in \mathcal{U}^{*}$ for all $s \in \operatorname{split}(p)$. We will assume that $B_{s} \in \mathcal{U}$ for all $s \in \operatorname{split}(p)$ (in the other case we work with $\omega \backslash \dot{B})$ ). Let $s_{0}$ be the stem of $p$. We will define a strategy $\sigma$ for player I in $\mathcal{H}(\mathcal{U}, \mathcal{F})$ as follows:

1. I starts by playing $W_{0}=B_{s_{0}}$.

2. Assume that player II plays $z_{0} \in\left[W_{0}\right]^{<\omega}$. Let $l_{0}=\max \left(z_{0}\right)$, player I will play $p_{0}=\bigcup\left\{p_{s_{0}} \mid t \in F_{i}^{s_{0}} \wedge i>l_{0}\right\}$. Note that $p_{0} \Vdash$ " $z_{0} \subseteq \dot{B}$ ".

3. Assume that player II plays $n_{0} \in \omega$. Now, the player I will play the set $W_{1}=\bigcap\left\{B_{s} \backslash l_{0} \mid s \in \operatorname{split}\left(p_{0}\right) \cap T_{0}\right\}$ (where $\left.T_{0}=T\left(p_{0}, n_{0}\right)\right)$.

4. In general, lets assume in the game it has already been played the sequence $\left\langle W_{0}, z_{0}, p_{0}, n_{0}, W_{1} \ldots W_{m}, z_{m}, p_{m}\right\rangle$. At the same time, player I has been making sure that the sequence $\left\langle p_{i}\right\rangle_{i \leq m}$ has the following properties:

(a) $p_{i+1} \leq_{T_{i}} p_{i}\left(\right.$ where $\left.T_{i}=T\left(p_{i}, n_{i}\right)\right)$ for all $i<m$.

(b) $p_{i} \Vdash$ " $z_{i} \subseteq \dot{B}$ " for all $i \leq m$. 
Now, assume that player II plays $n_{m} \in \omega$. Player I proceeds to play $W_{m+1}=$ $\bigcap\left\{B_{s} \backslash l_{m} \mid s \in \operatorname{split}\left(p_{m}\right) \cap T_{m}\right\}$ where $l_{m}=\max \left(z_{m}\right)$. Assume player II responds with $z_{m+1} \in\left[W_{m+1}\right]^{<\omega}$. For every $t \in T_{m} \cap \operatorname{split}\left(p_{m}\right)$, let $p_{m}^{t} \leq p_{m}$ be the biggest subtree such that stem $\left(p_{m}^{t}\right)=t$ and every node in $p_{m}^{t} \cap T_{m}$ is contained in $t$. Let $q^{t}=\bigcup\left\{\left(p_{m}^{t}\right)_{x} \mid x \in F_{i}^{t} \wedge i>l_{m+1}\right\}$ (where $l_{m+1}=\max \left(z_{m+1}\right)$ ) and now player I counterattacks with $p_{m+1}=\bigcup\left\{q^{t} \mid t \in T_{m} \cap \operatorname{split}\left(p_{m}\right)\right\}$. It is easy to see that $p_{m+1} \leq_{T_{m}} p_{m}$ and $p_{m+1} \Vdash$ " $z_{m+1} \subseteq \dot{B}$ ".

Since $\mathcal{F}$ is $\mathcal{U}$-Canjar, we know that $\sigma$ is not a winning strategy. Consider a run of the game where player I followed the strategy $\sigma$, but player II was the winner. In this way, we know that $U=\bigcup_{i \in \omega} z_{i} \in \mathcal{U}$ and $q=\bigcup_{i \in \omega} T_{i}$ is a condition of $\mathbb{P T}(\mathcal{F})$. By construction, it follows that $q \Vdash$ " $U \subseteq \dot{B}$ " and we are done.

\section{$5 \quad$ A model of $\omega_{1}=\mathfrak{u}<\mathfrak{s}$}

In order to increase the splitting number, it is enough to diagonalize an ultrafilter, and to preserve the ultrafilter number, it is enough preserve a $P$ point. In this way, in order to construct a model of $\mathfrak{u}<\mathfrak{s}$ it is enough to find a $P$-point $\mathcal{W}$ and an ultrafilter $\mathcal{U}$ that is $\mathcal{W}$-Canjar. In this situation, we will have that $\mathbb{P T}(\mathcal{W})$ adds an unsplit real while preserving $\mathcal{W}$. In this section, we will use our results to build a model of $\mathfrak{u}<\mathfrak{s}$. This result is not new, as it already holds in the Blass-Shelah model (see [7] or [3]). At least in the opinion of the authors, the combinatorics involved in our forcing are simpler than the ones from the Blass-Shelah forcing.

We will first focus on constructing a $\mathcal{B}$-Canjar ultrafilter for some $\mathfrak{b}$-family $\mathcal{B}$. Such ultrafilters can either be constructed under the Continuum Hypothesis or forced with a $\sigma$-closed forcing (see [8, [15, [17, or [28). This follows by the result of Shelah and the decomposition representation of Brendle and Raghavan. In 28 the first author, Michael Hrušák and Arturo Antonio Martinez CelisRodriguez published a proof of the consistency of $\mathfrak{b}<\mathfrak{s}$ and $\mathfrak{b}<\mathfrak{a}$ using directly the representation of Brendle and Raghavan. This section and the following borrows some of the arguments from [28.

Given $X$ a collection of finite non-empty subsets of $\omega$, we define $\mathcal{C}(X)=$ $\{A \subseteq \omega \mid \forall s \in X(s \cap A \neq \emptyset)\}$. The following lemma contains some of the combinatorial properties of compact sets that we will need:

Lemma 44 Let $\mathcal{F}$ be a filter, $\mathcal{D} \subseteq \mathcal{F}$ be a compact set and $X \in\left(\mathcal{F}^{<\omega}\right)^{+}$.

1. $\mathcal{C}(X)$ is a compact set.

2. There is $Y \in[X]^{<\omega}$ such that for every $A \in \mathcal{D}$ there is $s \in Y$ such that $s \subseteq A$. 
3. If $\mathcal{C}(X) \subseteq \mathcal{F}$ then for every $n \in \omega$ there is $S \in[X]^{<\omega}$ such that if $A_{0}, \ldots, A_{n} \in \mathcal{C}(S)$ and $F \in \mathcal{D}$ then $A_{0} \cap \ldots \cap A_{n} \cap F \neq \emptyset$.

4. If $\mathcal{U}$ is an ultrafilter and $Y \subseteq[\omega]^{<\omega}$ then $Y \in\left(\mathcal{U}^{<\omega}\right)^{+}$if and only if $\mathcal{C}(Y) \subseteq \mathcal{U}$.

Proof. For item 1, it is easy to see that $\mathcal{C}(X)$ is a closed subset of $\wp(\omega)$. We now prove item 2 , let $X \in\left(\mathcal{F}^{<\omega}\right)^{+}$and $\mathcal{D} \subseteq \mathcal{F}$ a compact set. For every $s \in X$, we define $U(s)=\{A \mid s \subseteq A\}$. It is easy to see that $U(s)$ is an open set and $\{U(s) \mid s \in X\}$ is an open cover for $\mathcal{D}$ (because $\left.X \in\left(\mathcal{F}^{<\omega}\right)^{+}\right)$. Since $\mathcal{D}$ is compact, there is $Y \in[X]^{<\omega}$ such that $\{U(s) \mid s \in Y\}$ is an open cover for $\mathcal{D}$. Clearly $Y$ is the set we were looking for.

We now prove 3 , let $\mathcal{C}(X) \subseteq \mathcal{F}$ and $n \in \omega$. Given $s \in X$ define $K(s)$ as the set of all $\left(A_{0}, \ldots, A_{n}\right) \in \mathcal{C}(s)^{n+1}$ with the property that there is $F \in \mathcal{D}$ such that $A_{0} \cap \ldots \cap A_{n} \cap F=\emptyset$. It is easy to see that $K(s)$ is a compact. Note that if $\left(A_{0}, \ldots, A_{n}\right) \in \bigcap_{s \in X} K(s)$ then $A_{0}, \ldots, A_{n} \in \mathcal{C}(X) \subseteq \mathcal{F}$ and there would be $F \in \mathcal{D} \subseteq \mathcal{F}$ such that $A_{0} \cap \ldots \cap A_{n} \cap F=\emptyset$ which is clearly a contradiction. Since the $K(s)$ are compact, then there must be $S \in[F]^{<\omega}$ such that $\bigcap_{s \in S} K(s)=\emptyset$. It is easy to see that this is the $S$ we are looking for.

We now prove 4 . Let $\mathcal{U}$ be an ultrafilter and $Y \subseteq[\omega]^{<\omega}$. We will prove that $Y \notin\left(\mathcal{U}^{<\omega}\right)^{+}$if and only if $\mathcal{C}(Y) \nsubseteq \mathcal{U}$. First assume that $Y \notin\left(\mathcal{U}^{<\omega}\right)^{+}$, this means that there is $A \in \mathcal{U}$ that does not contain any element of $Y$, so $B=\omega \backslash A$ intersects every element of $Y$, hence $B \in \mathcal{C}(Y)$ which implies that $\mathcal{C}(Y) \nsubseteq \mathcal{U}$. Now assume that $\mathcal{C}(Y) \nsubseteq \mathcal{U}$, so there is $B \in \mathcal{C}(Y)$ such that $B \notin \mathcal{U}$, hence $A=\omega \backslash B \in \mathcal{U}$. Since $B \in \mathcal{C}(Y)$, this implies that $A$ does not contain any element of $Y$, so $Y \notin\left(\mathcal{U}^{<\omega}\right)^{+}$.

We will need the following notion:

Definition 45 Let $\mathcal{I}$ be an ideal on $\omega$. We define $\mathbb{F}_{\sigma}(\mathcal{I})$ as the collection of all $F_{\sigma}$-filters $\mathcal{F}$ such that $\mathcal{F} \cap \mathcal{I}=\emptyset$. We order $\mathbb{F}_{\sigma}(\mathcal{I})$ by inclusion.

Note that an $F_{\sigma}$-filter $\mathcal{F}$ is in $\mathbb{F}_{\sigma}(\mathcal{I})$ if and only if $\mathcal{F} \cup \mathcal{I}^{*}$ generates a filter. The following are some properties of this types of forcings:

Lemma 46 Let $\mathcal{I}$ be an ideal on $\omega$.

1. $\mathbb{F}_{\sigma}(\mathcal{I})$ is a $\sigma$-closed forcing.

2. $\mathbb{F}_{\sigma}(\mathcal{I})$ adds an ultrafilter (which we will denote by $\mathcal{U}_{\text {gen }}(\mathcal{I})$ ) disjoint from $\mathcal{I}$.

3. $\mathbb{F}_{\sigma}(\mathcal{I}) * \mathbb{P} \mathbb{T}\left(\dot{\mathcal{U}}_{\text {gen }}(\mathcal{I})\right)$ and $\mathbb{F}_{\sigma}(\mathcal{I}) * \mathbb{M}\left(\dot{\mathcal{U}}_{\text {gen }}(\mathcal{I})\right)$ are proper forcings that destroy $\mathcal{I}$. 
If $\mathcal{A}$ is a MAD family, we will denote $\mathbb{F}_{\sigma}(\mathcal{A})$ instead of $\mathbb{F}_{\sigma}(\mathcal{I}(\mathcal{A}))$ and $\mathcal{U}_{\text {gen }}(\mathcal{A})$ instead of $\mathcal{U}_{\text {gen }}(\mathcal{I}(\mathcal{A}))$. Note that $\mathbb{F}_{\sigma}\left([\omega]^{<\omega}\right)$ is the collection of all $F_{\sigma}$-filters. In this case, we will only denote it by $\mathbb{F}_{\sigma}$ and by $\mathcal{U}_{\text {gen }}$ we will denote the generic ultrafilter added by $\mathbb{F}_{\sigma}$. The following lemma is easy and left to the reader:

Lemma 47 If $\mathcal{I}$ is an ideal, $X \subseteq[\omega]^{<\omega}$ and $\mathcal{F} \in \mathbb{F}_{\sigma}(\mathcal{I})$, then $\mathcal{F} \Vdash$ " $X \in$ $\left(\dot{\mathcal{U}}_{\text {gen }}(\mathcal{I})^{<\omega}\right)^{+}$" if and only if $\mathcal{C}(X) \subseteq\left\langle\mathcal{F} \cup \mathcal{I}^{*}\right\rangle$ (where $\left\langle\mathcal{F} \cup \mathcal{I}^{*}\right\rangle$ is the filter generated by $\left.\mathcal{F} \cup \mathcal{I}^{*}\right)$.

In particular, if $\mathcal{F} \in \mathbb{F}_{\sigma}$ and $X \subseteq[\omega]^{<\omega}$, then $\mathcal{F} \Vdash$ " $X \in\left(\dot{\mathcal{U}}_{\text {gen }}{ }^{<\omega}\right)^{+}$" if and only if $\mathcal{C}(X) \subseteq \mathcal{F}$. We will now prove the following:

Proposition 48 If $\mathcal{B} \in V$ is a $\mathfrak{b}$-family, then $\mathbb{F}_{\sigma}$ forces that $\dot{\mathcal{U}}_{\text {gen }}$ is $\mathcal{B}$-Canjar.

Proof. By the previous observation and since $\mathbb{F}_{\sigma}$ is $\sigma$-closed, it is enough to show that if $\mathcal{F} \Vdash " \bar{X}=\left\langle X_{n}\right\rangle_{n \in \omega} \subseteq\left(\dot{\mathcal{U}}_{\text {gen }}^{<\omega}\right)^{+}$" then there is $\mathcal{G} \leq \mathcal{F}$ and $f \in \mathcal{B}$ such that $\mathcal{C}\left(\bar{X}_{f}\right) \subseteq \mathcal{G}$.

Let $\mathcal{F}=\bigcup \mathcal{C}_{n}$ where each $\mathcal{C}_{n}$ is compact and they form an increasing chain. By a previous lemma, there is $g: \omega \longrightarrow \omega$ such that if $n \in \omega, F \in \mathcal{C}_{n}$ and $A_{0}, \ldots, A_{n} \in \mathcal{C}\left(X_{n} \cap \wp(g(n))\right)$ then $A_{0} \cap \ldots \cap A_{n} \cap F \neq \emptyset$. Since $\mathcal{B}$ is unbounded, then there is $f \in \mathcal{B}$ such that $f \not^{*} g$. We claim that $\mathcal{F} \cup \mathcal{C}\left(\bar{X}_{f}\right)$ generates a filter. Let $F \in \mathcal{C}_{n}$ and $A_{0}, \ldots, A_{m} \in \mathcal{C}\left(\bar{X}_{f}\right)$. We must show that $A_{0} \cap \ldots \cap A_{m} \cap F \neq \emptyset$. Since $f$ is not bounded by $g$, we may find $r>n, m$ such that $f(r)>g(r)$. In this way, $A_{0}, \ldots, A_{n} \in \mathcal{C}\left(X_{n} \cap \wp(g(n))\right)$ and then $A_{0} \cap \ldots \cap A_{m} \cap F \neq \emptyset$. Finally, we can define $\mathcal{G}$ as the filter generated by $\mathcal{F} \cup \mathcal{C}\left(\bar{X}_{f}\right)$.

In this way, we conclude the following:

Corollary 49 The forcing $\mathbb{F}_{\sigma} * \mathbb{P} \mathbb{T}\left(\dot{\mathcal{U}}_{\text {gen }}\right)$ is proper, adds an unsplit real, preserves all $\mathfrak{b}$-scales from the ground model and does not add Cohen reals.

A forcing notion is called weakly $\omega^{\omega}$-bounding if it does not add dominating reals. Unlike the $\omega^{\omega}$-bounding property, the weakly $\omega^{\omega}$-bounding property is not preserved under two step iteration (see [1]). However, Shelah proved the following preservation result:

Proposition 50 (Shelah, see [1]) If $\gamma \leq \omega_{2}$ is limit and $\left\langle\mathbb{P}_{\alpha}, \dot{\mathbb{Q}}_{\alpha} \mid \alpha \leq \gamma\right\rangle$ is a countable support iteration of proper forcings and each $\mathbb{P}_{\alpha}$ is weakly $\omega^{\omega}$ bounding (over $V$ ) then $\mathbb{P}_{\gamma}$ is weakly $\omega^{\omega}$-bounding. 
Note that $\mathbb{P}$ is weakly $\omega^{\omega}$-bounding if and only if it preserves the unboundedness of all (one) dominating families. By applying the result of Shelah we can easily conclude the following result.

Corollary 51 If $V$ satisfies $C H$ and $\left\langle\mathbb{P}_{\alpha}, \dot{\mathbb{Q}}_{\alpha} \mid \alpha \leq \omega_{2}\right\rangle$ is a countable support iteration of proper forcings such that $\mathbb{P}_{\alpha}$ forces that $\dot{\mathbb{Q}}_{\alpha}$ preserves the unboundedness of all well-ordered unbounded families, then $\mathbb{P}_{\omega_{2}}$ is weakly $\omega^{\omega}$-bounding.

With these results we can conclude the following result of Shelah:

Proposition 52 (Shelah) There is a model of $\omega_{1}=\mathfrak{b}<\mathfrak{s}=\operatorname{cov}(\mathcal{M})=\mathfrak{c}=$ $\omega_{2}$.

Proof. We perform a countable support iteration $\left\langle\mathbb{P}_{\alpha}, \dot{\mathbb{Q}}_{\alpha} \mid \alpha \leq \omega_{2}\right\rangle$ where $\mathbb{P}_{\alpha} \Vdash " \dot{\mathbb{Q}}_{\alpha}=\mathbb{F}_{\sigma} * \mathbb{M}\left(\dot{\mathcal{U}}_{\text {gen }}\right) "$. The result follows by the previous results.

Recall that a forcing $\mathbb{P}$ is almost $\omega^{\omega}$-bounding if for every $\mathbb{P}$-name $\dot{f}$ for an element of $\omega^{\omega}$ and $q \in \mathbb{P}$, there is $g \in \omega^{\omega}$ such that for every $A \in[\omega]^{\omega}$, there is $q_{A} \leq q$ such that $q_{A} \Vdash " g \uparrow A \mathbb{E}^{*} \dot{f} \uparrow A$ " (the reader may consult 2] to learn more about this property). It follows by the results of Shelah and Brendle and Raghavan that the iterands in the previous model are almost $\omega^{\omega}$ bounding, but it is not clear that this follows from our approach. In 19 it was proved that a forcing $\mathbb{P}$ is almost $\omega^{\omega}$-bounding if and only if it preserves every unbounded family of the ground model. The referee asked us if preserving the unboundedness of all well-ordered unbounded families imply the almost $\omega^{\omega}$ bounding property, we do not know the answer to this question.

As was mentioned before, by the result of Brendle and Raghavan, $\mathbb{F}_{\sigma} *$ $\mathbb{M}\left(\dot{\mathcal{U}}_{\text {gen }}\right)$ is forcing equivalent to the original creature forcing of Shelah for $\mathfrak{b}<\mathfrak{s}$. We will now prove that if we iterate $\mathbb{F}_{\sigma} * \mathbb{P T}\left(\dot{\mathcal{U}}_{\text {gen }}\right)$, we will get a model of $\mathfrak{u}<\mathfrak{s}$. Although we will not need the following result, it is illustrative to prove first the following:

Proposition 53 If $\mathcal{U}$ is a $P$-point and $\mathcal{F}$ is an $F_{\sigma}$-filter, then $\mathcal{F}$ is $\mathcal{U}$-Canjar.

Proof. Let $\mathcal{U}$ be a $P$-point and $\mathcal{F}=\bigcup_{n \in \omega} \mathcal{C}_{n}$ be an $F_{\sigma}$-filter, where $\left\langle\mathcal{C}_{n}\right\rangle_{n \in \omega}$ is an increasing sequence of compact sets. We will argue by contradiction, so assume that $\mathcal{F}$ is not $\mathcal{U}$-Canjar, i.e. player I has a winning strategy for the game $\mathcal{H}(\mathcal{U}, \mathcal{F})$, call $\sigma$ such strategy. We will use $\sigma$ to construct a winning strategy for I in the $P$-point game, which will obviously entail a contradiction.

Given $X \in\left(\mathcal{F}^{<\omega}\right)^{+}$and $n \in \omega$, choose $Y(X, n) \in[X]^{<\omega}$ such that every element of $\mathcal{C}_{n}$ contains an element of $Y(X, n)$ (which is possible by lemma 44). We now define $\pi$ a strategy for player I in $\mathcal{G}_{P \text {-point }}(\mathcal{U})$ as follows: 
1. Player I starts by playing $W_{0}=\sigma(\emptyset)$ (i.e. $W_{0}$ is the first play in the game $\mathcal{H}(\mathcal{U}, \mathcal{F}))$.

2. Assume player II plays $z_{0} \in\left[W_{0}\right]^{<\omega}$ as her response in $\mathcal{H}(\mathcal{U}, \mathcal{F})$. Let $p_{0}=\sigma\left(W_{0}, z_{0}\right)$ and $s_{0}$ be the stem of $p_{0}$. Define $n_{0}>d^{-1}\left(s_{0}\right)$ to be the least integer such that $d^{-1}\left(s_{0} \frown t\right)<n_{0}$ for all $t \in Y\left(\operatorname{spsuc}_{p_{0}}\left(s_{0}\right), 0\right)$. Player I will play (in $\left.\mathcal{G}_{P \text {-point }}(\mathcal{U})\right) W_{1}=\sigma\left(W_{0}, z_{0}, p_{0}, n_{0}\right)$ (i.e. his response in $\mathcal{H}(\mathcal{U}, \mathcal{F})$ if player II had played $\left.n_{0}\right)$.

3. In general assume that it has been played the sequence $\left\langle W_{0}, z_{0}, \ldots, W_{m}\right\rangle$. At the same time, in secret the player I has been constructed a partial play $\left\langle W_{0}, z_{0}, p_{0}, n_{0}, W_{1}, z_{1}, p_{1}, n_{1} \ldots, W_{m}\right\rangle$ in the game $\mathcal{H}(\mathcal{U}, \mathcal{F})$ following $\sigma$ such that for every $i<m$, the integer $n_{i}$ has the following property: for every $u \in T\left(p_{i}, n_{i-1}\right)$ (where $n_{-1}=d^{-1}\left(s_{0}\right)$ ) and for every $t \in Y\left(\operatorname{spsuc}_{p_{i}}(u), i\right)$, we have that $d^{-1}(u \frown t)<n_{i}$. Assume that player II plays $z_{m}$ as her next response in $\mathcal{G}_{P \text {-point }}(\mathcal{U})$. Let $p_{m}$ be the tree defined as $\sigma\left(W_{0}, z_{0}, n_{0}, W_{1}, \ldots, W_{m}, z_{m}\right)$ and let $n_{m}>n_{m-1}$ the least integer with the following property: for every $u \in T\left(p_{m}, n_{m-1}\right)$ and for every $t \in Y\left(\operatorname{spsuc}_{p_{m}}(u), m\right)$, we have that $d^{-1}(u \smile t)<n_{m}$. Player I will play the set $W_{m+1}$ that is defined as $\sigma\left(W_{0}, z_{0}, n_{0}, W_{1}, \ldots, W_{m}, z_{m}, p_{m}, n_{m}\right)$.

The game $\mathcal{G}_{P \text {-point }}(\mathcal{U})$ :

\begin{tabular}{|l|l|l|l|l|l|}
\hline I & $W_{0}$ & & $W_{1}$ & & $\ldots$ \\
\hline II & & $z_{0}$ & & $z_{1}$ & \\
\hline
\end{tabular}

The game $\mathcal{H}(\mathcal{U}, \mathcal{F})$ :

\begin{tabular}{|l|l|l|l|l|l|l|l|l|l|}
\hline $\mathrm{I}$ & $W_{0}$ & & $p_{0}$ & & $W_{1}$ & & $p_{1}$ & & $\ldots$ \\
\hline $\mathrm{II}$ & & $z_{0}$ & & $n_{0}$ & & $z_{1}$ & & $n_{1}$ & \\
\hline
\end{tabular}

We claim that $\pi$ is a winning strategy for player I in $\mathcal{G}_{P \text {-point }}(\mathcal{U})$. Consider a run of the game in which player I played according to $\pi$. Let $Z=\bigcup_{n \in \omega} z_{n}$, we will prove that $Z \notin \mathcal{U}$. Let $q=\bigcup_{i \in \omega} T\left(p_{i}, n_{i}\right)$ be the tree that was constructed during the play. It is easy to see that $q \in \mathbb{P} \mathbb{T}(\mathcal{F})$, but since player I was following his winning strategy $\sigma$ in the side game, we know that he won, so it must be the case that $Z \notin \mathcal{U}$. This shows that $\pi$ is a winning strategy for player I in $\mathcal{G}_{P \text {-point }}(\mathcal{U})$. Since player I can not have a winning strategy in the $P$-point game, we get a contradiction.

We will now prove that the forcing $\mathbb{F}_{\sigma} * \mathbb{P} \mathbb{T}\left(\dot{\mathcal{U}}_{\text {gen }}\right)$ preserves all ground model $P$-points. First we will need the following lemma, which is a slight generalization of part of lemma 44. 
Lemma 54 Let $\mathcal{F}$ be a filter, $\mathcal{D} \subseteq \mathcal{F}$ a compact set and $X_{1}, \ldots, X_{n} \subseteq[\omega]^{<\omega}$ such that $\mathcal{C}\left(X_{1}\right), \ldots, \mathcal{C}\left(X_{n}\right) \subseteq \mathcal{F}$. There are $Y_{1} \in\left[X_{1}\right]^{<\omega}, \ldots, Y_{n} \in\left[X_{n}\right]^{<\omega}$ such that for every $F \in \mathcal{D}$ and for every $A_{i}^{1}, \ldots, A_{i}^{n} \in \mathcal{C}\left(Y_{i}\right)$ (with $i \leq n$ ), we have that $F \cap \bigcap_{i, j \leq n} A_{i}^{j} \neq \emptyset$.

Proof. Consider the space $Z=\left(\prod_{i=1}^{n} \wp(\omega)^{n}\right) \times \mathcal{D}$, which we know is compact. Given $l \in \omega$, let $K(l)$ be the set of all $\left(\left\langle A_{i}^{1}, \ldots, A_{i}^{n}\right\rangle_{i \leq n}, F\right) \in Z$ such that $A_{i}^{1}, \ldots, A_{i}^{n} \in \mathcal{C}\left(X_{i} \cap \wp(l)\right)$ (for every $i \leq n$ ) and $F \cap \bigcap_{i, j \leq n} A_{i}^{j}=\emptyset$. Clearly $K(l)$ is a closed subspace. Since $\mathcal{C}\left(X_{1}\right), \ldots, \mathcal{C}\left(X_{n}\right), \mathcal{D} \subseteq \mathcal{F}$, we conclude that $\bigcap K(l)=\emptyset$, hence by the compactness of $Z$, we conclude that there is $l \in \omega$ $l \in \omega$

such that $K(l)=\emptyset$. Let $Y_{i}=X_{i} \cap \wp(l)$. It is clear that these are the sets we were looking for.

We can now prove the following result, which is a fusion of the proofs of proposition 53 and proposition 48 .

Proposition 55 If $\mathcal{W}$ is a P-point, then $\mathbb{F}_{\sigma}$ forces that $\dot{\mathcal{U}}_{\text {gen }}$ is $\mathcal{W}$-Canjar.

Proof. We will prove the proposition by contradiction. Assume there is $\mathcal{F}$ an $F_{\sigma}$-filter and $\sigma$ such that $\mathcal{F}$ forces that $\sigma$ is a winning strategy for player $I$ in $\mathcal{H}\left(\mathcal{W}, \dot{\mathcal{U}}_{\text {gen }}\right)$. Note that strategies for I are countable objects and since $\mathbb{F}_{\sigma}$ is $\sigma$ closed, it is enough to consider ground model strategies. Let $\mathcal{F}=\bigcup_{n \in \omega} \mathcal{C}_{n}$ where $\left\langle\mathcal{C}_{n}\right\rangle_{n \in \omega}$ is an increasing sequence of compact sets. We will use $\sigma$ to construct a winning strategy for I in the game $\mathcal{G}_{P \text {-point }}(\mathcal{W})$, which will be a contradiction.

Note that if $p$ is a Miller tree such that $p$ is a possible response of player I according to $\sigma$ and $s \in \operatorname{split}(p)$, then $\mathcal{F} \Vdash$ "spsuc $(s) \in\left(\dot{\mathcal{U}}_{\text {gen }}^{<\omega}\right)^{+}$" (this is because $\mathcal{F}$ is forcing that $\sigma$ is a strategy for player I, which implies that $p$ must be a legal move), in particular $\mathcal{C}\left(\operatorname{spsuc}_{p}(s)\right) \subseteq \mathcal{F}$.

For every $\mathcal{X}=\left\{X_{1}, \ldots, X_{n}\right\}$ such that $X_{i} \subseteq[\omega]^{<\omega} \backslash\{\emptyset\}$ and $\mathcal{C}\left(X_{i}\right) \subseteq \mathcal{F}$ for every $i \leq n$ and for every $k \in \omega$, fix a function $F_{(\mathcal{X}, k)}: \mathcal{X} \longrightarrow\left[\left[\omega^{<\omega]}\right]\right]^{<\omega}$ with the following properties:

1. $Y_{i}=F_{(\mathcal{X}, k)}\left(X_{i}\right) \in\left[X_{i}\right]^{<\omega}$ for every $i \leq n$.

2. For every $B \in \mathcal{C}_{k}$ and for every $A_{i}^{1}, \ldots, A_{i}^{n} \in \mathcal{C}\left(Y_{i}\right)$ (with $i \leq n$ ), we have that $B \cap \bigcap_{i, j \leq n} A_{i}^{j} \neq \emptyset$.

We know such $F_{(\mathcal{X}, k)}$ exists by lemma 54 . The proof now proceeds in a very similar way as the proof of proposition 53 . We define $\pi$ a strategy for player I in $\mathcal{G}_{P \text {-point }}(\mathcal{W})$ as follows:

1. Player I starts by playing $W_{0}=\sigma(\emptyset)$. 
2. Assume player II plays $z_{0} \in\left[W_{0}\right]^{<\omega}$. Let $p_{0}=\sigma\left(W_{0}, z_{0}\right), s_{0}$ be the stem of $p_{0}$ and $\mathcal{X}_{0}=\left\{\operatorname{spsuc}_{p_{0}}\left(s_{0}\right)\right\}$. Define $n_{0}>d^{-1}\left(s_{0}\right)$ to be the least integer such that $d^{-1}\left(s_{0}-t\right)<n_{0}$ for all $t \in F_{\left(\mathcal{X}_{0}, 0\right)}\left(\operatorname{spsuc}_{p_{0}}\left(s_{0}\right)\right)$. Player I will play (in $\left.\mathcal{G}_{P \text {-point }}(\mathcal{W})\right) W_{1}=\sigma\left(W_{0}, z_{0}, p_{0}, n_{0}\right)$.

3. In general assume that it has been played the sequence $\left\langle W_{0}, z_{0}, \ldots, W_{m}\right\rangle$. At the same time, secretly the player I has been constructing a sequence $\left\langle W_{0}, z_{0}, p_{0}, n_{0}, W_{1}, z_{1}, p_{1}, n_{1} \ldots, W_{m}\right\rangle$ that is being forced to be a partial play of the game $\mathcal{H}\left(\mathcal{W}, \dot{\mathcal{U}}_{\text {gen }}\right)$ following $\sigma$, such that for every $i<m$, the integer $n_{i}$ has the following property: letting $\mathcal{X}_{i}$ to be the set defined as $\left\{\operatorname{spsuc}_{p_{i}}(u) \mid u \in T\left(p_{i}, n_{i-1}\right)\right\}$ (where $n_{i-1}=d^{-1}\left(s_{0}\right)$ ), for every $t \in$ $F_{\left(\mathcal{X}_{i}, i\right)}\left(\operatorname{spsuc}_{p_{i}}(u)\right)$, we have that $d^{-1}(u \frown t)<n_{i}$. Assume that player II plays $z_{m}$ as her next response in $\mathcal{H}\left(\mathcal{W}, \dot{\mathcal{U}}_{\text {gen }}\right)$. Let $p_{m}$ be the tree given by $\sigma\left(W_{0}, z_{0}, n_{0}, W_{1}, \ldots, W_{m}, z_{m}\right)$ and let $n_{m}>n_{m-1}$ be the least integer with the following property: letting $\mathcal{X}_{m}=\left\{\operatorname{spsuc}_{p_{m}}(u) \mid u \in T\left(p_{m}, n_{m-1}\right)\right\}$, for every $t \in F_{\left(\mathcal{X}_{m}, m\right)}\left(\operatorname{spsuc}_{p_{m}}(u)\right)$, we have that $d^{-1}(u \frown t)<n_{m}$. Player I will play $W_{m+1}=\sigma\left(W_{0}, z_{0}, n_{0}, W_{1}, \ldots, W_{m}, z_{m}, p_{m}, n_{m}\right)$.

The game $\mathcal{G}_{\text {P-point }}(\mathcal{W})$ :

\begin{tabular}{|l|l|l|l|l|l|}
\hline $\mathrm{I}$ & $W_{0}$ & & $W_{1}$ & & $\ldots$ \\
\hline $\mathrm{II}$ & & $z_{0}$ & & $z_{1}$ & \\
\hline
\end{tabular}

The game $\mathcal{H}\left(\mathcal{W}, \dot{\mathcal{U}}_{\text {gen }}\right)$ :

\begin{tabular}{|l|l|l|l|l|l|l|l|l|l|}
\hline $\mathrm{I}$ & $W_{0}$ & & $p_{0}$ & & $W_{1}$ & & $p_{1}$ & & $\ldots$ \\
\hline $\mathrm{II}$ & & $z_{0}$ & & $n_{0}$ & & $z_{1}$ & & $n_{1}$ & \\
\hline
\end{tabular}

We claim that $\pi$ is a winning strategy for player I in $\mathcal{G}_{P \text {-point }}(\mathcal{W})$. Consider a run of the game in which player I played according to $\pi$. Let $Z=\bigcup_{n \in \omega} z_{n}$, we will prove that $Z \notin \mathcal{U}$. Let $q=\bigcup_{i \in \omega} T\left(p_{i}, n_{i}\right)$ be te tree that was constructed by player I during the play. It is easy to see that $\mathcal{F} \cup\left\{\mathcal{C}\left(\operatorname{spsuc}_{q}(s)\right) \mid s \in \operatorname{split}(q)\right\}$ generates an $F_{\sigma}$-filter, call it $\mathcal{K}$. Note that $\mathcal{K} \leq \mathcal{F}$ hence $\mathcal{K}$ forces that $\sigma$ is a winning strategy for player I in $\mathcal{H}\left(\mathcal{W}, \dot{\mathcal{U}}_{\text {gen }}\right)$. Moreover, $\mathcal{K}$ forces that $q \in$ $\mathbb{P T}\left(\dot{\mathcal{U}}_{\text {gen }}\right)$. Since player I is forced to win in $\mathcal{H}\left(\mathcal{W}, \dot{\mathcal{U}}_{\text {gen }}\right)$, it must be the case that $Z \notin \mathcal{W}$. This shows that $\pi$ is a winning strategy for player I in $\mathcal{G}_{P \text {-point }}(\mathcal{W})$. Since player I can not have a winning strategy in the $P$-point game, we get a contradiction.

In this way, we conclude that $\mathbb{F}_{\sigma} * \mathbb{P} \mathbb{T}\left(\dot{\mathcal{U}}_{\text {gen }}\right)$ preserves all ground model $P$-points. Note that after forcing with $\mathbb{F}_{\sigma} * \mathbb{P} \mathbb{T}\left(\dot{\mathcal{U}}_{\text {gen }}\right)$, there are intermediate extensions with $P$-points that are not preserved $\left(\mathcal{U}_{\text {gen }}\right.$ for example), however, all 
ground model $P$-points are preserved. By iterating $\mathbb{F}_{\sigma} * \mathbb{P} \mathbb{T}\left(\dot{\mathcal{U}}_{\text {gen }}\right)$ with countable support, we get the following result [7]:

Corollary 56 (Blass-Shelah) The inequality $\mathfrak{u}<\mathfrak{s}$ is consistent with ZFC.

In [48] Mildenberger proved the following interesting result:

Proposition 57 (Mildenberger) It is consistent that there is a proper forcing that diagonalizes an ultrafilter and preserves a P-point.

Note that our work provides an alternative proof of the theorem of Mildenberger.

We would like to mention that in original model of Shelah of $\mathfrak{b}<\mathfrak{s}$, in the Blass-Shelah model, and in our model, the almost disjointness number is equal to $\omega_{1}$. In [58] (using also the results from [15]) it is proved that $\mathfrak{a}=\omega_{1}$ after iterating (with countable support) the forcing $\mathbb{F}_{\sigma} * \mathbb{M}\left(\dot{\mathcal{U}}_{\text {gen }}\right)$. A similar approach works when iterating $\mathbb{F}_{\sigma} * \mathbb{P T}\left(\dot{\mathcal{U}}_{\text {gen }}\right)$. It is also possible to use the technique of theorem 6.6 in 52 to show that $\diamond(\mathfrak{b})$ holds in that model, hence $\mathfrak{a}=\omega_{1}$ (see 52] for the definition of $\diamond(\mathfrak{b})$ and the proof that $\diamond(\mathfrak{b})$ implies $\left.\mathfrak{a}=\omega_{1}\right)$. Since this result will not be used in the paper, we omit the details.

Regarding the groupwise-density number $\mathfrak{g}$, it can be proved that $\mathfrak{g}=\omega_{2}$ holds in our model. In particular, this is a model of $\mathfrak{u}<\mathfrak{g}$, so the Near Coherence of Filters principle holds in our model. This was proved by David Chodounský, Jonathan Verner and the first author and will be published in a consequent paper. The reader may consult [5] to lear nore about $\mathfrak{g}$ and the Near Coherence of filters principle.

\section{A model of $\omega_{1}=\mathfrak{u}<\mathfrak{a}$}

In this section, we will prove that every MAD family can be destroyed with a proper forcing that preserves $P$-points, answering the questions of Brendle and Shelah. First, we will prove that if $\mathcal{A}$ is a MAD family, then $\dot{\mathcal{U}}_{\text {gen }}(\mathcal{A})$ is forced to be $\mathcal{B}$-Canjar for every $\mathfrak{b}$-family $\mathcal{B}$ in the ground model. In [15] and 28 it is proved that after adding $\omega_{1}$-Cohen reals, $\mathbb{F}_{\sigma}(\mathcal{A})$ forces that $\mathcal{U}_{\text {gen }}(\mathcal{A})$ has these properties. Obviously, we can not use these results since we do not want to add Cohen reals. In this section, we will prove that the Cohen reals were really not needed in the first place. This proof takes inspiration in the proof of $\mathfrak{b}<\mathfrak{a}$ by Brendle in [8]. When the authors were preparing the paper, they learned from Zdomskyy that he has found a different proof that the preliminary Cohen reals are not needed. 
Definition 58 We say a MAD family $\mathcal{A}$ is a Laflamme family if $\mathcal{I}(\mathcal{A})$ can not be extended to an $F_{\sigma}$ ideal (or equivalently, $\mathcal{I}(\mathcal{A})^{*}$ can not be extended to an $F_{\sigma}$-filter).

Laflamme proved that the Continuum Hypothesis implies that there is a Laflamme MAD family. Minami and Sakai constructed a Laflamme MAD family assuming $\mathfrak{p}=\mathfrak{c}$. It is a major open problem if ZFC implies the existence of Laflamme MAD families. The reader may consult [42] and [51] for more information on Laflamme MAD families.

Note that if $\mathcal{A}$ is not Laflamme (i.e. $\mathcal{A}$ can be extended to an $F_{\sigma}$-ideal), then $\mathbb{F}_{\sigma} * \mathbb{P} \mathbb{T}\left(\dot{\mathcal{U}}_{\text {gen }}\right)$ destroys $\mathcal{A}$ below some condition, in this way, we only need to take care of Laflamme families. The following is a simple lemma that will be needed later:

Lemma 59 Let $\mathcal{A}$ be a $M A D$ family and $\mathcal{F} \in \mathbb{F}_{\sigma}(\mathcal{A})$. If there is a proper forcing $\mathbb{P}$ such that $\mathbb{P}$ forces the following statement: "There is $\mathcal{D} \in[\mathcal{A}]^{\omega}$ such that $\mathcal{I}(\mathcal{A})^{*} \subseteq\langle\mathcal{F} \cup\{\omega \backslash A \mid A \in \mathcal{D}\}\rangle$ " then $\mathcal{A}$ is not Laflamme.

Proof. Since $\mathbb{P}$ is a proper forcing, we can find a condition $p \in \mathbb{P}$ and $\mathcal{D}_{1} \in$ $[\mathcal{A}]^{\omega}$ in $V$ such that $p \Vdash$ " $\mathcal{D} \subseteq \mathcal{D}_{1}$ ". It is then easy to see that $\mathcal{I}(\mathcal{A})^{*} \subseteq$ $\left\langle\mathcal{F} \cup\left\{\omega \backslash A \mid A \in \mathcal{D}_{1}\right\}\right\rangle$ so $\mathcal{A}$ is not Laflamme.

Given $X \subseteq[\omega]^{<\omega}$ and $A \in[\omega]^{\omega}$, we define $\operatorname{Catch}(X, A)=\{s \in X \mid s \subseteq A\}$. We will need the following definition:

Definition 60 Let $\mathcal{F}$ be an $F_{\sigma}$-filter, $X \subseteq[\omega]^{<\omega}$ and $A \in[\omega]^{\omega}$. We will say that $\star(\mathcal{F}, X, A)$ holds, if the following conditions are satisfied:

1. $A \in \mathcal{F}^{+}$.

2. If $B \in[A]^{\omega} \cap \mathcal{F}^{+}$then $C a t c h(X, B) \in\left(\mathcal{F}^{<\omega}\right)^{+}$(i.e. for every $F \in \mathcal{F}$ there is $s \in X$ such that $s \subseteq F \cap B)$.

Let $\mathcal{A}$ be a MAD family, $\mathcal{F} \in \mathbb{F}_{\sigma}(\mathcal{A})$ and $X \subseteq[\omega]^{<\omega}$ such that $\mathcal{C}(X) \subseteq$ $\left\langle\mathcal{F} \cup \mathcal{I}(\mathcal{A})^{*}\right\rangle$. Fix $\left\langle\mathcal{C}_{n}\right\rangle_{n \in \omega}$ an increasing family of compact sets such that $\mathcal{F}=$ $\cup \mathcal{C}_{n}$. The Brendle game $\mathcal{B} \mathcal{R}(\mathcal{A}, \mathcal{F}, X)$ is defined as follows,

\begin{tabular}{|l|l|l|l|l|l|l|l}
\hline $\mathrm{I}$ & $Y_{0}$ & & $Y_{1}$ & & $Y_{2}$ & & $\cdots$ \\
\hline $\mathrm{II}$ & & $s_{0}$ & & $s_{1}$ & & $s_{2}$ & $\cdots$ \\
\hline
\end{tabular}

${ }^{8}$ This game was based on the rank arguments used by Brendle in [8]. A similar (but different) approach using games was used by Brendle and Taylor in [17. 
Where $Y_{m} \in \mathcal{I}(\mathcal{A})^{*}, s_{m} \in\left[Y_{m}\right]^{<\omega}$ intersects all the elements of $\mathcal{C}_{m}$ and $\max \left(s_{m}\right)<\min \left(s_{m+1}\right) \unlhd$ Player I wins the game if $\bigcup_{n \in \omega} s_{n}$ contains an element of $X$. Note that this is an open game for I, i.e., if she wins, then she wins already in a finite number of steps. By the Gale-Stewart theorem (see [39]), the Brendle game is determined. We will now prove the following:

Proposition 61 Let $\mathcal{A}$ be a Laflamme $M A D$ family and $\mathcal{F} \in \mathbb{F}_{\sigma}(\mathcal{A})$. For every family $\left\{X_{n} \mid n \in \omega\right\}$ such that $\mathcal{C}\left(X_{n}\right) \subseteq\left\langle\mathcal{F} \cup \mathcal{I}(\mathcal{A})^{*}\right\rangle$, there is a countable family $\mathcal{D} \in[\mathcal{A}]^{\omega}$ such that $\star\left(\mathcal{F}, A, X_{n}\right)$ holds for every $n \in \omega$ and $A \in \mathcal{D}$.

Proof. By $V\left[C_{\alpha}\right]$ we denote an extension of $V$ by adding $\alpha$-Cohen reals (the reader should not be worried by the use of Cohen reals in the proof, see the paragraph after this result for more information). We first claim the following:

Claim 62 If $X \subseteq[\omega]^{<\omega}$ is such that $\mathcal{C}(X) \subseteq\left\langle\mathcal{F} \cup \mathcal{I}(\mathcal{A})^{*}\right\rangle$, then in $V\left[C_{\omega_{1}}\right]$ the player I has a winning strategy for $\mathcal{B R}(\mathcal{A}, \mathcal{F}, X)$.

We will prove the claim by contradiction, since $\mathcal{B R}(\mathcal{A}, \mathcal{F}, X)$ is determined, we assume that II has a winning strategy, call it $\pi$. We will choose a tree $T \subseteq$ $\left([\omega]^{<\omega}\right)^{<\omega}$ and a family $\left\{B_{t} \mid t \in T\right\} \subseteq \mathcal{I}(\mathcal{A})^{*}$ with the following properties:

1. $\emptyset \in T$ and $B_{\emptyset}=\omega$ (this is just a technical step).

2. If $t=\left\langle s_{0}, s_{1}, \ldots, s_{n}\right\rangle \in T$ then $\left\langle B_{\left\langle s_{0}\right\rangle}, s_{0}, B_{\left\langle s_{0}, s_{1}\right\rangle}, s_{1}, \ldots, B_{\left\langle s_{0}, s_{1}, \ldots, s_{n}\right\rangle}, s_{n}\right\rangle$ is a legal partial play of $\mathcal{B R}(\mathcal{A}, \mathcal{F}, X)$ in which Player II is using her strategy $\pi$.

An important remark is in order here: Note that for example, for every $s \in[\omega]^{<\omega}$ there may be infinitely many $B \in \mathcal{I}(\mathcal{A})^{*}$ such that $\langle B, s\rangle$ is a legal partial play of $\mathcal{B R}(\mathcal{A}, \mathcal{F}, X)$ in which Player II is using her strategy $\pi$. For $B_{\langle s\rangle}$ we just choose and fix one of them. The tree $T$ and $\left\{B_{t} \mid t \in T\right\}$ are recursively constructed as follows:

1. $\emptyset \in T$ and $B_{\emptyset}=\omega$.

2. $T_{1}$ is the set of all $\langle s\rangle$ such that $s \in[\omega]^{<\omega}$ and there is $B \in \mathcal{I}(\mathcal{A})^{*}$ for which $\langle B, s\rangle$ is a legal partial play of $\mathcal{B R}(\mathcal{A}, \mathcal{F}, X)$ in which Player II is using her strategy $\pi$.

3. For every $s$ such that $\langle s\rangle \in T_{1}$, we choose $B_{s} \in \mathcal{I}(\mathcal{A})^{*}$ for which $\left\langle B_{s}, s\right\rangle$ is a legal partial play.

\footnotetext{
${ }^{9}$ Note that the game $\mathcal{B R}(\mathcal{A}, \mathcal{F}, X)$ does not only depend on $\mathcal{F}$, but on its representation as an increasing union of compact sets. A more formal notation would be $\mathcal{B R}\left(\mathcal{A},\left\langle\mathcal{C}_{n}\right\rangle_{n \in \omega}, X\right)$.
} 
4. Given a node $t=\left\langle s_{0}, s_{1}, \ldots, s_{n}\right\rangle \in T$ (and we know that the sequence $\left\langle B_{\left\langle s_{0}\right\rangle}, s_{0}, B_{\left\langle s_{0}, s_{1}\right\rangle}, s_{1}, \ldots, B_{\left\langle s_{0}, s_{1}, \ldots, s_{n}\right\rangle}, s_{n}\right\rangle$ is a legal partial play) let $\operatorname{suc}_{T}(t)$ be the set of all $z \in[\omega]^{<\omega}$ for which there is $B \in \mathcal{I}(\mathcal{A})^{*}$ such that $\left\langle B_{\left\langle s_{0}\right\rangle}, s_{0}, B_{\left\langle s_{0}, s_{1}\right\rangle}, s_{1}, \ldots, B_{\left\langle s_{0}, s_{1}, \ldots, s_{n}\right\rangle}, s_{n}, B, z\right\rangle$ is a legal partial play (in which Player II is using her strategy $\pi$ ). We fix $B_{t}-\langle z\rangle \in \mathcal{I}(\mathcal{A})^{*}$ with this property.

Note that if $t=\left\langle s_{0}, s_{1}, \ldots, s_{n}\right\rangle \in T$, then $\bigcup_{i \leq n} s_{i}$ does not contain an element of $X$, this is because $\pi$ is a winning strategy for player II. Clearly $T$ is a countable tree with no isolated branches, so it is equivalent to Cohen forcing when viewed as a forcing notion. Since $T$ is countable, it appears in an intermediate extension of $V\left[C_{\omega_{1}}\right]$. Let $\beta<\omega_{1}$ such that $T \in V\left[C_{\beta}\right]$.

Given $Y \in \mathcal{I}(\mathcal{A})^{*}$ define the set $D_{Y}$ of all $t=\left\langle s_{0}, s_{1}, \ldots, s_{n}\right\rangle \in T$ such that there is $i \leq n$ for which $s_{i} \subseteq Y$. It is easy to see that each $D_{Y}$ is an open dense subset of $T$. Let $G \in V\left[C_{\omega_{1}}\right]$ be a $\left(T, V\left[C_{\beta}\right]\right)$-generic branch through $T$. It is easy to see that $G$ induces a legal play of the game in which II followed her strategy. Let $D=\bigcup G$, and since $\pi$ is a winning strategy for II, we conclude that $D$ does not contain an element of $X$. By genericity $D \in\left\langle\mathcal{I}(\mathcal{A})^{*} \cup \mathcal{F}\right\rangle^{+}$however, $\omega \backslash D \in \mathcal{C}(X) \subseteq\left\langle\mathcal{I}(\mathcal{A})^{*} \cup \mathcal{F}\right\rangle$ which is obviously a contradiction. This finishes the proof of the claim.

We work in $V\left[C_{\omega_{1}}\right]$, where player I has winning strategies for all of the games $\mathcal{B R}\left(\mathcal{A}, \mathcal{F}, X_{n}\right)$ with $n \in \omega$. Let $\pi_{n}$ be the winning strategy for the game $\mathcal{B R}\left(\mathcal{A}, \mathcal{F}, X_{n}\right)$. Let $\mathcal{W}$ be set of elements of $\mathcal{I}(\mathcal{A})^{*}$ that may be played by I following her winning strategy in any of these games. It is not hard to see that $\mathcal{W}$ is countable. Note that if $W \in \mathcal{W}$ then $W$ almost contains every element of $\mathcal{A}$ except for finitely many (this is because $\left.W \in \mathcal{I}(\mathcal{A})^{*}\right)$. Let $\mathcal{A}^{\prime} \subseteq \mathcal{A}$ be the set of all $A \in \mathcal{A}$ for which there is $W \in \mathcal{W}$ such that $A \nsubseteq^{*} W$. Note that $\mathcal{A}^{\prime}$ is countable. Since $\mathcal{A}$ is Laflamme in $V$, by a previous lemma, $\mathcal{I}(\mathcal{A})^{*}$ it is not contained in $\left\langle\mathcal{F} \cup\left\{\omega \backslash B \mid B \in \mathcal{A}^{\prime}\right\}\right\rangle$, so there is $A_{0} \in \mathcal{A}$ such that $\omega \backslash A_{0} \notin\left\langle\mathcal{F} \cup\left\{\omega \backslash B \mid B \in \mathcal{A}^{\prime}\right\}\right\rangle$. This implies that $A_{0} \in \mathcal{F}^{+}$and $A_{0}$ is almost contained in every member of $\mathcal{W}$. We claim that $\star\left(\mathcal{F}, A_{0}, X_{n}\right)$ holds for each $n \in \omega$. Let $B \in \wp\left(A_{0}\right) \cap \mathcal{F}^{+}$we will now show that $\operatorname{Catch}\left(X_{n}, B\right)$ is positive for each $n \in \omega$. Let $F \in \mathcal{F}$ and consider the following play in $\mathcal{B R}\left(\mathcal{A}, \mathcal{F}, X_{n}\right)$,

\begin{tabular}{|l|l|l|l|l|l|l|l}
\hline $\mathrm{I}$ & $W_{0}$ & & $W_{1}$ & & $W_{2}$ & & $\cdots$ \\
\hline $\mathrm{II}$ & & $s_{0}$ & & $s_{1}$ & & $s_{2}$ & $\cdots$ \\
\hline
\end{tabular}

where the $W_{i}$ are played by I according to $\pi_{n}, s_{i} \in[B \cap F]^{<\omega}$ and intersects every element of $\mathcal{C}_{i}$. This is possible since $B \cap F$ is positive and is almost contained in every $W_{n}$. Since $\pi_{n}$ is a winning strategy, this means that I wins the game, which entails that $\bigcup s_{n} \subseteq B \cap F$ contains an element of $X_{n}$. We can then obtain each $A_{n+1}$ by repeating the same argument and using that 
$\mathcal{I}(\mathcal{A})^{*}$ it is not contained in $\left\langle\mathcal{F} \cup\left\{\omega \backslash B \mid B \in \mathcal{A}^{\prime}\right\} \cup\left\{\omega \backslash A_{0}, . ., \omega \backslash A_{n}\right\}\right\rangle$. Let $\mathcal{D}_{1}=\left\{A_{n} \mid n \in \omega\right\}$.

We know that $V\left[C_{\omega_{1}}\right] \models \star\left(\mathcal{F}, A_{n}, X_{m}\right)$ for every $n, m \in \omega$. However, it is easy to see that the statement $\star\left(\mathcal{F}, A_{n}, X_{m}\right)$ is absolute between models of ZFC (in fact, we only need that it is downwards absolute, which is easy). So $V \models \star\left(\mathcal{F}, A_{n}, X_{m}\right)$ for every $n, m \in \omega$. Since $\mathbb{C}_{\omega_{1}}$ has the countable chain condition, there is $\mathcal{D} \in[\mathcal{A}]^{\omega}$ such that $\mathbb{C}_{\omega_{1}} \Vdash$ " $\mathcal{D}_{1} \subseteq \mathcal{D}$ ". By the previous remark, we may assume that that $\star\left(\mathcal{F}, A, X_{n}\right)$ holds for every $n \in \omega$ and $A \in \mathcal{D}$.

The reader might feel that we cheated in the previous proof by adding the Cohen reals, and sincerely, we have, but it was a "legal cheating". We only used the Cohen reals to find ground model objects, and after finding them, we came back to the ground model as if nothing happened.

Given $A \in[\omega]^{\omega}$ and $l \in \omega$ define $\operatorname{Part}_{l}(A)$ as the set of all sequences $\left\langle B_{1}, \ldots, B_{l}\right\rangle$ such that $A=\bigcup_{i \leq l} B_{i}$ and $B_{i} \cap B_{j}=\emptyset$ whenever $i \neq j$. Note that $\operatorname{Part}_{l}(A)$ is a compact space with the natural topology. Also it is clear that if $A \in \mathcal{F}^{+}$and $\left\langle B_{1}, \ldots, B_{l}\right\rangle \in \operatorname{Part}_{l}(A)$ then there is $j \leq l$ such that $B_{j} \in \mathcal{F}^{+}$.

Lemma 63 Let $\mathcal{F}$ be a filter, $\mathcal{C} \subseteq \mathcal{F}$ a compact set and $X \in\left(\mathcal{F}^{<\omega}\right)^{+}$. Let $A$ such that $\star(A, \mathcal{F}, X)$ holds and let $l \in \omega$. There is $n \in \omega$ with the property that for all $\left\langle B_{1}, \ldots, B_{l}\right\rangle \in \operatorname{Part}_{l}(A)$ there is $i \leq l$ such that if $F \in \mathcal{C}$ then $X \cap \wp\left(B_{i} \cap n\right)$ contains a subset of $F$.

Proof. Let $U_{n}$ be the set of all $\left\langle B_{1}, \ldots, B_{l}\right\rangle \in \operatorname{Part}_{l}(A)$ such that there is $i \leq l$ with the property that if $F \in \mathcal{C}$ then $X \cap \wp\left(B_{i} \cap n\right)$ contains a subset of $F$. Note that $\left\{U_{n} \mid n \in \omega\right\}$ is an open cover (recall that $\star(A, \mathcal{F}, X)$ holds and if we split $A$ into finitely many pieces, then one of the pieces must be in $\mathcal{F}^{+}$) and the result follows since $\operatorname{Part}_{l}(A)$ is compact.

We will now prove the following:

Proposition 64 Let $\mathcal{F}$ be a filter, $\mathcal{C} \subseteq \mathcal{F}$ a compact set, $X \in\left(\mathcal{F}^{<\omega}\right)^{+}, A \in$ $[\omega]^{\omega}$ such that $\star(A, \mathcal{F}, X)$ holds and $l \in \omega$. There is $Y \in[X]^{<\omega}$ such that if $C_{1}, \ldots, C_{l} \in \mathcal{C}(Y)$ and $F \in \mathcal{C}$ then there is $s \in Y \cap[A]^{<\omega}$ such that $s \subseteq$ $C_{1} \cap \ldots \cap C_{l} \cap F$.

Proof. Let $n$ such that for every $\left\langle B_{1}, \ldots, B_{2^{l}}\right\rangle \in \operatorname{Part}_{2^{l}}(A)$ and for every $F \in \mathcal{C}$ there is $j \leq 2^{l}$ for which $X \cap \wp\left(B_{j} \cap n\right)$ contains a subset of $F$. Let $Y=X \cap \wp(l)$, we will see that $Y$ has the desired properties. Let $C_{1}, \ldots, C_{l} \in \mathcal{C}(Y)$ and $F \in \mathcal{C}$. For every $s: l \longrightarrow 2$ define $B_{s}$ as the set of all $a \in A$ such that $a \in C_{i}$ if and only if $s(i)=1$. Clearly $\left\langle B_{s}\right\rangle_{s \in 2^{l}} \in \operatorname{Part}_{2^{l}}(A)$ and we may conclude that there is $s$ such that $Y \cap \wp\left(B_{s} \cap n\right)$ contains an element of $F$. Since $C_{1}, \ldots, C_{l} \in \mathcal{C}(Y)$ we conclude that $s$ must be the constant function 1, and this entails the desired conclusion.

We can then finally conclude the following: 
Proposition 65 If $\mathcal{A}$ is a Laflamme $M A D$ family, then $\mathbb{F}_{\sigma}(\mathcal{A})$ forces that $\dot{\mathcal{U}}_{\text {gen }}(\mathcal{A})$ is $\mathcal{B}$-Canjar for every $\mathfrak{b}$-family $\mathcal{B}$ in the ground model.

Proof. It is enough to show that if $\mathcal{F} \Vdash " \bar{X}=\left\langle X_{n}\right\rangle_{n \in \omega} \subseteq\left(\dot{\mathcal{U}}_{\text {gen }}(\mathcal{A})^{<\omega}\right)^{+}$" then there is $\mathcal{G} \leq \mathcal{F}$ and $f \in \mathcal{B}$ such that $\mathcal{C}\left(\bar{X}_{f}\right) \subseteq \mathcal{G}$. Let $\mathcal{F}=\bigcup \mathcal{C}_{n}$ where each $\mathcal{C}_{n}$ is compact and they form an increasing chain. By the previous results, we may find $\left\{A_{n} \mid n \in \omega\right\} \subseteq \mathcal{A}$ such that $\star\left(A_{n}, \mathcal{F}, X_{m}\right)$ holds for every $n, m \in \omega$. We can then find an increasing function $g: \omega \longrightarrow \omega$ such that the following holds:

*) For every $n \in \omega$ and for every $i \leq n$, if $Y=X \cap \wp(g(n))$ then for every $C_{0}, \ldots, C_{n} \in \mathcal{C}(Y)$ and $F \in \mathcal{C}_{n}$ there is $s \in Y \cap\left[A_{i}\right]^{<\omega}$ such that $s \subseteq$ $C_{0} \cap \ldots \cap C_{l} \cap F$.

Since $\mathcal{B}$ is unbounded, we can find $f \in \mathcal{B}$ that is not dominated by $g$. It is easy to see that $\mathcal{G}=\left\langle\mathcal{F} \cup \mathcal{C}\left(\bar{X}_{f}\right)\right\rangle$ is a condition in $\mathbb{F}_{\sigma}(\mathcal{A})$ and has the desired properties.

We can conclude:

Corollary 66 Every MAD family can be destroyed with a forcing that is proper, adds an unsplit real, preserves all $\mathfrak{b}$-families from the ground model and does not add Cohen reals.

Proof. If $\mathcal{A}$ is not Laflamme, then it can be destroyed by $\mathbb{F}_{\sigma} * \mathbb{P T}\left(\dot{\mathcal{U}}_{g e n}\right)$ (below some condition). If $\mathcal{A}$ is Laflamme, then we can destroy it with $\mathbb{F}_{\sigma}(\mathcal{A}) *$ $\mathbb{P} \mathbb{T}\left(\dot{\mathcal{U}}_{\text {gen }}(\mathcal{A})\right)$.

By iterating the forcings in the previous corollary, we get the following:

Proposition 67 (Shelah) There is a model of $\omega_{1}=\mathfrak{b}<\mathfrak{a}=\mathfrak{s}=\mathfrak{c}=\omega_{2}$.

Note that if we iterate with countable support forcings of the type $\mathbb{F}_{\sigma}(\mathcal{A}) *$ $\mathbb{M}\left(\dot{\mathcal{U}}_{\text {gen }}(\mathcal{A})\right)$ and $\mathbb{F}_{\sigma} * \mathbb{M}\left(\dot{\mathcal{U}}_{\text {gen }}\right)$, we will get a model of $\omega_{1}=\mathfrak{b}<\mathfrak{a}=\mathfrak{s}=$ $\operatorname{cov}(\mathcal{M})=\mathfrak{c}=\omega_{2}$. In order to preserve $P$-points, we must use the Miller forcing instead of the Mathias forcing, as we are going to show now. We will prove that for every MAD family $\mathcal{A}$, the forcing $\mathbb{F}_{\sigma}(\mathcal{A}) * \mathbb{P} \mathbb{T}\left(\dot{\mathcal{U}}_{\text {gen }}(\mathcal{A})\right)$ preserves all ground model $P$-points. We will need the following generalization of lemma 63 .

Lemma 68 Let $l \in \omega, \mathcal{F}$ a filter, $\mathcal{D} \subseteq \mathcal{F}$ a compact set, $X_{1}, \ldots, X_{n} \subseteq[\omega]^{<\omega} \backslash\{\emptyset\}$ such that $\mathcal{C}\left(X_{1}\right), \ldots, \mathcal{C}\left(X_{n}\right) \subseteq \mathcal{F}$ and $A \in[\omega]^{\omega}$ such that $\star\left(A, \mathcal{F}, X_{i}\right)$ holds for every $i \leq n$. There is $m \in \omega$ such that for every $\left\langle B_{1}, \ldots, B_{l}\right\rangle \in$ Part $_{l}(A)$, there is $i \leq l$ such that for every $F \in \mathcal{D}$ and for every $k \leq n$, the set $\left(B_{i} \cap F\right) \cap m$ contains an element of $X_{k}$. 
Proof. Let $U_{m}$ be the set of all $\left\langle B_{1}, \ldots, B_{l}\right\rangle \in \operatorname{Part}_{l}(A)$ such that there is $i \leq l$ with the property that if $F \in \mathcal{C}$ and $k \leq n$ then, $X_{k} \cap \wp\left(B_{i} \cap m\right)$ contains a subset of $F$. Note that $\left\{U_{n} \mid n \in \omega\right\}$ is an open cover and the result follows since $\operatorname{Part}_{l}(A)$ is compact.

With this result, we can prove the following generalization of lemma 64:

Lemma 69 Let $\mathcal{F}$ be a filter, $\mathcal{D} \subseteq \mathcal{F}$ a compact set, $X_{1}, \ldots, X_{n} \subseteq[\omega]^{<\omega}$ such that $\mathcal{C}\left(X_{1}\right), \ldots, \mathcal{C}\left(X_{n}\right) \subseteq \mathcal{F}$ and $A \in[\omega]^{\omega}$ such that $\star\left(A, \mathcal{F}, X_{i}\right)$ holds for every $i \leq n$. There are $Y_{1} \in\left[X_{1}\right]^{<\omega}, \ldots, Y_{n} \in\left[X_{n}\right]^{<\omega}$ such that for every $F \in \mathcal{D}$ and for every $C_{i}^{1}, \ldots, C_{i}^{n} \in \mathcal{C}\left(Y_{i}\right)$ (with $i \leq n$ ), for every $k \leq n$ there is $s \in Y_{k} \cap[A]^{<\omega}$ such that $s \subseteq F \cap \bigcap_{i, j \leq n} C_{i}^{j}$.

Proof. Let $l=n^{2}$ and by lemma 68, we know there is $m \in \omega$ such that for every $\left\langle B_{1}, \ldots, B_{2^{l}}\right\rangle \in \operatorname{Part}_{2^{l}}(A)$ there is $i \leq 2^{l}$ such that for every $F \in \mathcal{D}$ and for every $k \leq n$, the set $\left(B_{i} \cap F\right) \cap m$ contains an element of $X_{k}$. Let $Y_{i}=X_{i} \cap \wp(m)$ for every $i \leq n$. We will see that the sets $Y_{1}, \ldots, Y_{n}$ have the desired properties. Let $C_{i}^{1}, \ldots, C_{i}^{n} \in \mathcal{C}\left(Y_{i}\right)$ (with $i \leq n$ ) and $F \in \mathcal{C}$. For every $s: n \times n \longrightarrow 2$ define $B_{s}$ as the set of all $a \in A$ such that $a \in C_{i}^{j}$ if and only if $s(i, j)=1$. Clearly $\left\langle B_{s}\right\rangle_{s \in 2^{l}} \in \operatorname{Part}_{2^{l}}\left(A_{l}\right)$ and we may conclude that there is $s: n \times n \longrightarrow 2$ such that $Y_{i} \cap \wp\left(B_{s} \cap m\right)$ contains an element of $F$ for every $i \leq n$. Since $C_{i}^{1}, \ldots, C_{i}^{n} \in \mathcal{C}\left(Y_{i}\right)$ we conclude that $s$ must be the constant function 1 , and this entails the desired conclusion.

We can now prove the following result, which is a combination of proposition 55 and proposition 65.

Proposition 70 If $\mathcal{W}$ is a P-point and $\mathcal{A}$ is a Laflamme MAD family, then $\mathbb{F}_{\sigma}(\mathcal{A})$ forces that $\dot{\mathcal{U}}_{\text {gen }}(\mathcal{A})$ is $\mathcal{W}$-Canjar.

Proof. We will prove the proposition by contradiction. Assume there is $\mathcal{F} \in$ $\mathbb{F}_{\sigma}(\mathcal{A})$ and $\sigma$ such that $\mathcal{F}$ forces that $\sigma$ is a winning strategy for player $I$ in $\mathcal{H}\left(\mathcal{W}, \dot{\mathcal{U}}_{\text {gen }}(\mathcal{A})\right)$. Since strategies for player I are countable objects and since $\mathbb{F}_{\sigma}(\mathcal{A})$ is $\sigma$-closed, it is enough to consider ground model strategies. Let $\mathcal{F}=$ $\bigcup_{n \in \omega} \mathcal{C}_{n}$ where $\left\langle\mathcal{C}_{n}\right\rangle_{n \in \omega}$ is an increasing sequence of compact sets. We will use $\sigma$ to construct a winning strategy for player I in the game $\mathcal{G}_{P \text {-point }}(\mathcal{W})$, which will be a contradiction.

Let $L$ be the collection of all $p_{s}$ such that $p$ is a possible response of player $I$ according to $\sigma$ and $s \in \operatorname{split}(p)$. In the same way as in the proof of proposition 55] if $p_{s} \in L$, then $\mathcal{F} \Vdash$ "spsuc $(s) \in\left(\dot{\mathcal{U}}_{\text {gen }}^{<\omega}\right)^{+}$", hence in particular $\mathcal{C}\left(\operatorname{spsuc}_{p}(s)\right) \subseteq\left\langle\mathcal{F} \cup \mathcal{I}(\mathcal{A})^{*}\right\rangle$. Since $L$ is countable we may assume (by extending $\mathcal{F}$ if necessary) that $\mathcal{C}\left(\operatorname{spsuc}_{p}(s)\right) \subseteq \mathcal{F}$ for every $p_{s} \in L$. By 61, we can find a family $\left\{A_{n} \mid n \in \omega\right\} \subseteq \mathcal{A}$ such that $\star\left(A_{n}, \mathcal{F}, \mathcal{C}\left(\operatorname{spsuc}_{p}(s)\right)\right)$ holds for every $n \in \omega$ and $p_{s} \in L$. 
For every $\mathcal{X}=\left\{X_{1}, \ldots, X_{n}\right\} \in\left[\left\{\operatorname{spsuc}_{p}(s) \mid p \in L \wedge s \in \operatorname{split}(p)\right\}\right]^{<\omega}$ and for every $k \in \omega$, fix a function $F_{(\mathcal{X}, k)}: \mathcal{X} \longrightarrow\left[\left[\omega^{<\omega}\right]\right]^{<\omega}$ with the following properties:

1. $Y_{i}=F_{(\mathcal{X}, k)}\left(X_{i}\right) \in\left[X_{i}\right]^{<\omega}$ for every $i \leq n$.

2. For every $B \in \mathcal{C}_{k}$, for every $C_{i}^{1}, \ldots, C_{i}^{n} \in \mathcal{C}\left(Y_{i}\right)$ (with $i \leq n$ ) and for every $k_{1}, k_{2} \leq n$, we have that $B \cap \bigcap_{i, j \leq n} C_{i}^{j}$ contains an element of $Y_{k_{1}} \cap\left[A_{k_{2}}\right]^{<\omega}$.

We know such $F_{(\mathcal{X}, k)}$ exists by the previous lemma. The proof now proceeds in a very similar way as the proof of 55 . We define $\pi$ a strategy for player I in $\mathcal{G}_{\text {P-point }}(\mathcal{W})$ as follows:

1. Player I starts by playing $W_{0}=\sigma(\emptyset)$.

2. Assume player II plays $z_{0} \in\left[W_{0}\right]^{<\omega}$. Let $p_{0}=\sigma\left(W_{0}, z_{0}\right), s_{0}$ be the stem of $p_{0}$ and $\mathcal{X}_{0}=\left\{\operatorname{spsuc}_{p_{0}}\left(s_{0}\right)\right\}$. Define $n_{0}>d^{-1}\left(s_{0}\right)$ to be the least integer such that $d^{-1}\left(s_{0} \frown t\right)<n_{0}$ for all $t \in F_{\left(\mathcal{X}_{0}, 0\right)}\left(\operatorname{spsuc}_{p_{0}}\left(s_{0}\right)\right)$. Player I will play (in $\left.\mathcal{G}_{P \text {-point }}(\mathcal{W})\right) W_{1}=\sigma\left(W_{0}, z_{0}, p_{0}, n_{0}\right)$.

3. In general, assume that it has been played the sequence $\left\langle W_{0}, z_{0}, \ldots, W_{m}\right\rangle$. At the same time, the player I has secretly been constructing a sequence $\left\langle W_{0}, z_{0}, p_{0}, n_{0}, W_{1}, z_{1}, p_{1}, n_{1} \ldots, W_{m}\right\rangle$ that is being forced to be a partial play of the game $\mathcal{H}\left(\mathcal{W}, \dot{\mathcal{U}}_{\text {gen }}(\mathcal{A})\right)$ following $\sigma$ such that for every $i<$ $m$, the integer $n_{i}$ has the following important property: letting $\mathcal{X}_{i}=$ $\left\{\operatorname{spsuc}_{p_{i}}(u) \mid u \in T\left(p_{i}, n_{i-1}\right)\right\}$ (we define $n_{-1}=d^{-1}\left(s_{0}\right)$ ), for every $t \in$ $F_{\left(\mathcal{X}_{i}, i\right)}\left(\operatorname{spsuc}_{p_{i}}(u)\right)$, we have that $d^{-1}(u \frown t)<n_{i}$. Assume that player II plays $z_{m}$ as her next response in $\mathcal{H}\left(\mathcal{W}, \dot{\mathcal{U}}_{\text {gen }}(\mathcal{A})\right)$. Let $p_{m}$ be the tree $\sigma\left(W_{0}, z_{0}, n_{0}, W_{1}, \ldots, W_{m}, z_{m}\right)$ and let $n_{m}>n_{m-1}$ the least integer with the following property: letting $\mathcal{X}_{m}=\left\{\operatorname{spsuc}_{p_{m}}(u) \mid u \in T\left(p_{m}, n_{m-1}\right)\right\}$, for every $t \in F_{\left(\mathcal{X}_{m}, m\right)}\left(\operatorname{spsuc}_{p_{m}}(u)\right)$, we have that $d^{-1}(u \frown t)<n_{m}$. Player I will play $W_{m+1}=\sigma\left(W_{0}, z_{0}, n_{0}, W_{1}, \ldots, W_{m}, z_{m}, p_{m}, n_{m}\right)$.

The game $\mathcal{G}_{\text {P-point }}(\mathcal{W})$ :

\begin{tabular}{|l|l|l|l|l|l|}
\hline $\mathrm{I}$ & $W_{0}$ & & $W_{1}$ & & $\ldots$ \\
\hline $\mathrm{II}$ & & $z_{0}$ & & $z_{1}$ & \\
\hline
\end{tabular}

The game $\mathcal{H}\left(\mathcal{W}, \dot{\mathcal{U}}_{\text {gen }}(\mathcal{A})\right)$ :

\begin{tabular}{|l|l|l|l|l|l|l|l|l|l|}
\hline $\mathrm{I}$ & $W_{0}$ & & $p_{0}$ & & $W_{1}$ & & $p_{1}$ & & $\ldots$ \\
\hline $\mathrm{II}$ & & $z_{0}$ & & $n_{0}$ & & $z_{1}$ & & $n_{1}$ & \\
\hline
\end{tabular}

We claim that $\pi$ is a winning strategy for player I in $\mathcal{G}_{P \text {-point }}(\mathcal{W})$. Consider a run of the game in which player I played according to $\pi$. Let $Z=\bigcup_{n \in \omega} z_{n}$, we 
will prove that $Z \notin \mathcal{U}$. Let $q=\bigcup_{i \in \omega} T\left(p_{i}, n_{i}\right)$ be the tree that was constructed by player I during the play. It is easy to see that $\mathcal{F} \cup\left\{\mathcal{C}\left(\operatorname{spsuc}_{q}(s)\right) \mid s \in \operatorname{split}(q)\right\}$ generates a condition of $\mathbb{F}_{\sigma}(\mathcal{A})$, call if $\mathcal{K}$. Note that $\mathcal{K} \leq \mathcal{F}$ hence $\mathcal{K}$ forces that $\sigma$ is a winning strategy for player I in $\mathcal{H}\left(\mathcal{W}, \dot{\mathcal{U}}_{\text {gen }}\right)$. Moreover, $\mathcal{K}$ forces that $q \in \mathbb{P T}\left(\dot{\mathcal{U}}_{\text {gen }}(\mathcal{A})\right)$. Since player I is forced to win in $\mathcal{H}\left(\mathcal{W}, \dot{\mathcal{U}}_{\text {gen }}(\mathcal{A})\right)$, it must be the case that $Z \notin \mathcal{W}$. This shows that $\pi$ is a winning strategy for player I in $\mathcal{G}_{P \text {-point }}(\mathcal{W})$. Since player I can not have a winning strategy in the $P$-point game, we get a contradiction.

We can now answer the questions of Brendle and Shelah:

Theorem 71 Every MAD family can be destroyed with a proper forcing that preserves all $P$-points from the ground model. In particular, it is consistent that $\omega_{1}=\mathfrak{u}<\mathfrak{a}=\omega_{2}$.

\section{MAD families build up from closed sets}

The closed almost disjointness number was introduced by Brendle and Khomskii in [14]. The invariant $\mathfrak{a}_{\text {closed }}$ is defined as the smallest number of closed sets of $[\omega]^{\omega}$ such that its union is a MAD family. Since singletons are closed, it follows that $\mathfrak{a}_{\text {closed }} \leq \mathfrak{a}$ and it is uncountable by a result of Mathias (see [4] or 61]). The following are some known results regarding this cardinal invariant:

\section{Proposition 72}

1. (Raghavan, Törnquist independently) $\mathfrak{p} \leq \mathfrak{a}_{\text {closed }}$ (see [61]).

2. (Brendle and Khomskii) It is consistent that $\mathfrak{a}_{\text {closed }}<\mathfrak{b}$ (see [14] and [15]).

3. (Brendle and Raghavan) It is consistent that $\mathfrak{b}<\mathfrak{a}_{\text {closed }}$ (see [15]).

4. (Raghavan and Shelah) $\mathfrak{d}=\omega_{1}$ implies $\mathfrak{a}_{\text {closed }}=\omega_{1}$ (see [54] and [15]).

There are still many interesting open questions regarding $\mathfrak{a}_{\text {closed }}$. The following problems are still open:

Problem 73 (Raghavan) Does $\mathfrak{h} \leq \mathfrak{a}_{\text {closed }}$ ?

Problem 74 (Brendle, Khomskii) Does $\mathfrak{s}=\omega_{1}$ imply $\mathfrak{a}_{\text {closed }}=\omega_{1}$ ?

The reader may consult [14 or 15] for more information and open problems regarding $\mathfrak{a}_{\text {closed }}$. If $\mathcal{D} \subseteq[\omega]^{\omega}$ is an $A D$ family, we denote its ortogonal $\mathcal{D}^{\perp}$ as the set of all $B \subseteq \omega$ that are almost disjoint with every element of $\mathcal{D}$. 
As mentioned before, Brendle and Raghavan proved that it is consistent that $\mathfrak{b}<\mathfrak{a}_{\text {closed }}$. In fact, they build two models in which this inequality holds. One is using the creature forcing of Shelah and another was using a c.c.c. forcing, similar to the model constructed in [8]. In both cases, their forcings add Cohen reals. We will combine their results with ours to build a model of $\mathfrak{b}<\mathfrak{a}_{\text {closed }}$ without adding Cohen reals, moreover; this inequality holds in the model constructed in the previous section. The key result is the following proposition, which was implicitly proved in the Lemma 7 of [15]:

Proposition 75 (Brendle, Raghavan) Let $\mathcal{D} \subseteq[\omega]^{\omega}$ be a closed $A D$ family. If $\mathcal{U}$ is a P-point such that $\mathcal{D} \cap \mathcal{U}=\emptyset$, then there is $U \in \mathcal{U} \cap \mathcal{D}^{\perp}$.

Proof. Note that if $\mathcal{D} \cap \mathcal{U}=\emptyset$ then $\mathcal{I}(\mathcal{D}) \cap \mathcal{U}=\emptyset$ since $\mathcal{U}$ is an ultrafilter. We argue by contradiction, assume that $\mathcal{U} \cap \mathcal{D}^{\perp}=\emptyset$. Note that this implies that for every $B \in \mathcal{U}$ there are $\left\{A_{n} \mid n \in \omega\right\} \subseteq \mathcal{D}$ such that $\left|B \cap A_{n}\right|=\omega$ for every $n \in \omega$. Consider the Laver forcing $\mathbb{L}(\mathcal{U})$. Since $\mathcal{I}(\mathcal{D})$ is an analytic set, by a result of Blass (see [4]), there is $p \in \mathbb{L}(\mathcal{U})$ such that either $[p] \subseteq \mathcal{I}(\mathcal{D})$ or $[p] \cap \mathcal{I}(\mathcal{D})=\emptyset$. Since $\mathcal{U}$ is a $P$-point, we can find $q \leq p$ and $U \in \mathcal{U}$ such that $\operatorname{suc}_{q}(s)={ }^{*} U$ for every $s \in q$ such that $s$ extends the stem of $q$.

Let $\left\{A_{n} \mid n \in \omega\right\} \subseteq \mathcal{D}$ such that $A_{n} \cap U$ is infinite for every $n \in \omega$. On one hand, we can find a branch $f_{0} \in[q]$ such that $i m\left(f_{0}\right) \subseteq A_{0}$, so $i m\left(f_{0}\right) \in \mathcal{I}(\mathcal{D})$, but on the other hand, we can find $f_{1} \in[q]$ such that $i m\left(f_{1}\right) \cap A_{n}$ is infinite for every $n \in \omega$, so $i m\left(f_{1}\right) \notin \mathcal{I}(\mathcal{D})$. These two statement are in contradiction since we know that either $[q] \subseteq \mathcal{I}(\mathcal{D})$ or $[q] \cap \mathcal{I}(\mathcal{D})=\emptyset$.

We can now conclude the following:

Corollary 76 Let $\mathcal{A}=\bigcup_{\alpha \in \kappa} \mathcal{C}_{\alpha}$ be a MAD family such that each $\mathcal{C}_{\alpha}$ is a closed subset of $[\omega]^{\omega}$. Let $\mathcal{U}$ be a P-point such that $\mathcal{A} \cap \mathcal{U}=\emptyset$. If $\mathbb{P}$ is a forcing that diagonalizes $\mathcal{U}$, and $G \subseteq \mathbb{P}$ is a generic filter, then $V[G] \models " \mathcal{A}^{V[G]}=\bigcup_{\alpha \in \kappa} \mathcal{C}_{\alpha}^{V[G]}$ is not a MAD family" (where $\mathcal{C}_{\alpha}^{V[G]}$ is the reinterpretation of $\mathcal{C}_{\alpha}$ in the model $V[G])$.

Proof. Let $G \subseteq \mathbb{P}$ be a generic filter. We argue in $V[G]$. Let $B \in[\omega]^{\omega}$ be a pseudointersection of $\mathcal{U}$. We claim that $B$ is almost disjoint with $\mathcal{A}^{V[G]}$. By the previous result, for every $\alpha<\kappa$ there is $U_{\alpha} \in \mathcal{U}$ such that the following statement holds in $V$ : " $U_{\alpha}$ is almost disjoint with every element of $\mathcal{C}_{\alpha}$ ". Since $\mathcal{C}_{\alpha}$ is a closed set, this is an absolute statement, so $U_{\alpha}$ is almost disjoint with every element of $\mathcal{C}_{\alpha}^{V[G]}$. Furthermore, since $B \subseteq^{*} U_{\alpha}$, then $B$ is also almost disjoint with every element of $\mathcal{C}_{\alpha}^{V[G]}$ for every $\alpha<\kappa$, hence $B$ is almost disjoint with $\mathcal{A}^{V[G]}$.

In 18 it was proved that Canjar ultrafilters are $P$-points (moreover, Canjar ultrafilters are precisely the "strong $P$-points", see $[6$ for the definition of strong $P$-point). In this way, we can conclude the following: 
Corollary 77 The following statements are consistent with the axioms of ZFC:

1. (Brendle, Raghavan) $\omega_{1}=\mathfrak{b}<\mathfrak{a}_{\text {closed }}=\operatorname{cov}(\mathcal{M})=\mathfrak{c}=\omega_{2}$.

2. $\omega_{1}=\mathfrak{u}<\mathfrak{a}_{\text {closed }}=\omega_{2}$.

\section{Open Problems}

We will list some open problems the authors do not know how to answer. Regarding the forcings $\mathbb{Q}(\mathcal{F})$ of Sabok and Zapletal, we know that they might or might not diagonalize $\mathcal{F}$. It would be interesting to know the answer of the following:

Problem 78 Is there a nice combinatorial characterization for the filters $\mathcal{F}$ for which $\mathbb{Q}(\mathcal{F})$ diagonalizes $\mathcal{F}$ ?

The forcings $\mathbb{M}(\mathcal{F})$ and $\mathbb{L}(\mathcal{F})$ have been proven to be very useful (see for example [38, [53, [21], 36, 22] or [13 for some applications of this forcings). We expect the forcings $\mathbb{P T}(\mathcal{F})$ to have interesting applications as well. It would then be useful to have a deeper understanding of such forcings. For example we have the following:

Problem 79 Let $\mathcal{F}$ be a filter.

1. Is there a combinatorial characterization of when $\mathbb{P T}(\mathcal{F})$ does not add Cohen reals?

2. Is there a combinatorial characterization of when $\mathbb{P T}(\mathcal{F})$ does not add dominating reals?

3. Is there a filter $\mathcal{F}$ such that $\mathbb{P} T(\mathcal{F})$ does not add dominating reals but $\mathbb{M}(\mathcal{F})$ adds dominating reals?

It would be interesting if the previous properties have characterizations in terms of the Katětov order, similar to the results for $\mathbb{Q}(\mathcal{F})$ obtained in [55]. Regarding the preservation of $P$-points, we have the following:

\section{Problem 80}

1. Let $\mathcal{U}$ be a P-point and $\mathcal{F}$ a Canjar filter such that $\mathbb{P} \mathbb{T}(\mathcal{F})$ forces that $\mathcal{U}$ generates a non-meager filter. Does $\mathbb{P} \mathbb{T}(\mathcal{F})$ preserve $\mathcal{U} 110$

2. Assuming $\mathrm{CH}$, is there a Canjar filter $\mathcal{F}$ such that $\mathbb{P T}(\mathcal{F})$ destroys all P-points?

\footnotetext{
${ }^{10}$ This question was recently answered positively by Chodounský, Verner and the first author. This result will be published in another article.
} 
Regarding half-Cohen reals, we do not know the answer of the following questions:

Problem 81 If $\mathbb{P}$ does not add half-Cohen reals and $\mathbb{P} \Vdash “ \dot{\mathbb{Q}}$ does not add halfCohen reals", is it true that $\mathbb{P} * \dot{\mathbb{Q}}$ does not add half-Cohen reals?

Problem 82 If $\delta$ is a limit ordinal, $\left\langle\mathbb{P}_{\alpha}, \dot{\mathbb{R}}_{\alpha} \mid \alpha<\delta\right\rangle$ is a countable support iteration of proper forcings such that each $\mathbb{P}_{\alpha}$ does not add half-Cohen reals, is it true that $\mathbb{P}_{\delta}$ does not add half-Cohen reals? What if each $\mathbb{P}_{\alpha}$ does not add dominating reals?

Recall that not adding Cohen reals is not preserved under two step iteration by the result of Zapletal in 63 .

Acknowledgement 83 The authors would like to thank Juris Steprāns for valuable comments and hours of stimulating conversations. We would also like to thank the referee for her/his generous comments that improved the paper. The research for this paper was started when the first author was a postdoc at York University, concluded after he became a postdoc in the University of Toronto, and the final version was finished while working for the Universidad Autónoma de México (UNAM). He would like to express his gratitude to the three institutions.

\section{References}

[1] Uri Abraham. Proper forcing. In Handbook of Set Theory, pages 333-394. Springer Netherlands, Dordrecht, 2010.

[2] Uri Abraham and Menachem Magidor. Cardinal arithmetic. In Handbook of set theory. Vols. 1, 2, 3, pages 1149-1227. Springer, Dordrecht, 2010.

[3] Tomek Bartoszyński and Haim Judah. Set theory: on the structure of the real line. Wellesley, MA: A. K. Peters Ltd., 1995.

[4] Andreas Blass. Selective ultrafilters and homogeneity. Ann. Pure Appl. Logic, 38(3):215-255, 1988.

[5] Andreas Blass. Combinatorial cardinal characteristics of the continuum. In Handbook of set theory. Vols. 1, 2, 3, pages 395-489. Springer, Dordrecht, 2010.

[6] Andreas Blass, Michael Hrušák, and Jonathan Verner. On strong P-points. Proc. Amer. Math. Soc., 141(8):2875-2883, 2013. 
[7] Andreas Blass and Saharon Shelah. There may be simple $P_{\aleph_{1}}-$ and $P_{\aleph_{2}}-$ points and the Rudin-Keisler ordering may be downward directed. Ann. Pure Appl. Logic, 33(3):213-243, 1987.

[8] Jörg Brendle. Mob families and mad families. Arch. Math. Logic, 37(3):183197, 1997.

[9] Jörg Brendle. Mad families and iteration theory. In Logic and algebra, volume 302 of Contemp. Math., pages 1-31. Amer. Math. Soc., Providence, RI, 2002.

[10] Jörg Brendle. The almost-disjointness number may have countable cofinality. Trans. Amer. Math. Soc., 355(7):2633-2649, 2003.

[11] Jörg Brendle. Mad families and ultrafilters. Acta Univ. Carolin. Math. Phys., 48(2):19-35, 2007.

[12] Jörg Brendle and Vera Fischer. Mad families, splitting families and large continuum. J. Symbolic Logic, 76(1):198-208, 2011.

[13] Jörg Brendle and Michael Hrušák. Countable fréchet boolean groups: An independence result. The Journal of Symbolic Logic, 74(3):1061-1068, 2009.

[14] Jörg Brendle and Yurii Khomskii. Mad families constructed from perfect almost disjoint families. J. Symbolic Logic, 78(4):1164-1180, 2013.

[15] Jörg Brendle and Dilip Raghavan. Bounding, splitting, and almost disjointness. Ann. Pure Appl. Logic, 165(2):631-651, 2014.

[16] Jörg Brendle and Shunsuke Yatabe. Forcing indestructibility of MAD families. Ann. Pure Appl. Logic, 132(2-3):271-312, 2005.

[17] Jörg Brendle and Andrew Brooke-Taylor. A variant proof of $\operatorname{Con}(\mathfrak{b}<\mathfrak{a})$. Proceedings of the 2014 RIMS meeting on Reflection principles and set theory of large cardinals, 042014.

[18] R. Michael Canjar. Mathias forcing which does not add dominating reals. Proc. Amer. Math. Soc., 104(4):1239-1248, 1988.

[19] David Chodounský, Dusan Repovs, and Lyubomyr Zdomskyy. Mathias forcing and combinatorial covering properties of filters. J. Symb. Log., 80(4):1398-1410, 2015.

[20] Alan Dow. On MAD families and sequential order. In Papers on general topology and applications (Amsterdam, 1994), volume 788 of Ann. New York Acad. Sci., pages 79-94. New York Acad. Sci., New York, 1996.

[21] Ilijas Farah. Semiselective coideals. Mathematika, 45(1):79-103, 1998.

[22] Ilijas Farah and Jindřich Zapletal. Four and more. Annals of Pure and Applied Logic, 140(1):3 - 39, 2006. Cardinal Arithmetic at work: the 8th Midrasha Mathematicae Workshop. 
[23] Vera Fischer, Sy D. Friedman, Diego A. Mejía, and Diana C. Montoya. Coherent systems of finite support iterations. J. Symb. Log., 83(1):208236, 2018.

[24] Vera Fischer and Bernhard Irrgang. Non-dominating ultrafilters. Acta Universitatis Carolinae. Mathematica et Physica, 051(Suppl):13-17, 2010.

[25] Vera Fischer and Diego Alejandro Mejía. Splitting, bounding, and almost disjointness can be quite different. Canad. J. Math., 69(3):502-531, 2017.

[26] Vera Fischer and Juris Steprāns. The consistency of $\mathfrak{b}=\kappa$ and $\mathfrak{s}=\kappa^{+}$. Fund. Math., 201(3):283-293, 2008.

[27] Vera Fischer and Asger Törnquist. Template iterations and maximal cofinitary groups. Fund. Math., 230(3):205-236, 2015.

[28] Osvaldo Guzmán, Michael Hrušák, and Arturo Martínez-Celis. Canjar filters II. Proceedings of the 2014 RIMS meeting on Reflection principles and set theory of large cardinals, pages 59-67, 2014.

[29] Osvaldo Guzmán, Michael Hrušák, and Arturo Martínez-Celis. Canjar filters. Notre Dame J. Form. Log., 58(1):79-95, 2017.

[30] Lorenz J. Halbeisen. Combinatorial set theory. With a gentle introduction to forcing. Berlin: Springer, 1nd edition edition, 2017.

[31] Rodrigo Hernández-Gutiérrez and Paul J. Szeptycki. Some observations on filters with properties defined by open covers. Commentationes Mathematicae Universitatis Carolinae, 56(3):355-364, 2015.

[32] Michael Hrušák. Combinatorics of filters and ideals. In Set theory and its applications, volume 533 of Contemp. Math., pages 29-69. Amer. Math. Soc., Providence, RI, 2011.

[33] Michael Hrušák. Almost disjoint families and topology. In Recent progress in general topology. III, pages 601-638. Atlantis Press, Paris, 2014.

[34] Michael Hrušák. Katětov order on Borel ideals. Archive for Mathematical Logic, 56(7):831-847, Nov 2017.

[35] Michael Hrušák and Salvador García Ferreira. Ordering MAD families a la Katětov. J. Symbolic Logic, 68(4):1337-1353, 2003.

[36] Michael Hrušák and Hiroaki Minami. Mathias-Prikry and Laver-Prikry type forcing. Ann. Pure Appl. Logic, 165(3):880-894, 2014.

[37] Michael Hrušák and Jindřich Zapletal. Forcing with quotients. Arch. Math. Logic, 47(7-8):719-739, 2008.

[38] M. Hrušák and U. A. Ramos-García. Malykhin's problem. Adv. Math., 262:193-212, 2014. 
[39] Alexander S. Kechris. Classical descriptive set theory, volume 156 of Graduate Texts in Mathematics. Springer-Verlag, New York, 1995.

[40] Miloš S. Kurilić. Cohen-stable families of subsets of integers. J. Symbolic Logic, 66(1):257-270, 2001.

[41] Claude Laflamme. Forcing with filters and complete combinatorics. Annals of Pure and Applied Logic, 42(2):125 - 163, 1989.

[42] Claude Laflamme. Zapping small filters. Proc. Amer. Math. Soc., 114(2):535-544, 1992.

[43] Richard Laver. Products of infinitely many perfect trees. J. London Math. Soc. (2), 29(3):385-396, 1984.

[44] A. R. D. Mathias. Happy families. Ann. Math. Logic, 12(1):59-111, 1977.

[45] Diego A. Mejía. Template iterations with non-definable ccc forcing notions. Ann. Pure Appl. Logic, 166(11):1071-1109, 2015.

[46] Diego Alejandro Mejía. Matrix iterations and Cichon's diagram. Arch. Math. Logic, 52(3-4):261-278, 2013.

[47] David Meza. Ideals and filters on countable sets. PhD thesis, Universidad Autónoma de México, 2009.

[48] Heike Mildenberger. Diagonalising an ultrafilter and preserving a $p$-point. to appear in Fundamenta Mathematicae.

[49] Heike Mildenberger. Forcing with $F_{\sigma^{-}}$and with summable filters. In Set theory: recent trends and applications, volume 17 of Quad. Mat., pages 183-194. Dept. Math., Seconda Univ. Napoli, Caserta, 2006.

[50] Arnold W. Miller. Rational perfect set forcing. In Axiomatic set theory (Boulder, Colo., 1983), volume 31 of Contemp. Math., pages 143-159. Amer. Math. Soc., Providence, RI, 1984.

[51] Hiroaki Minami and Hiroshi Sakai. Katětov and Katětov-Blass orders on $F_{\sigma}$-ideals. Arch. Math. Logic, 55(7-8):883-898, 2016.

[52] Justin Tatch Moore, Michael Hrušák, and Mirna Džamonja. Parametrized $\diamond$ principles. Trans. Amer. Math. Soc., 356(6):2281-2306, 2004.

[53] Dilip Raghavan. A model with no strongly separable almost disjoint families. Israel J. Math., 189:39-53, 2012.

[54] Dilip Raghavan and Saharon Shelah. Comparing the closed almost disjointness and dominating numbers. Fund. Math., 217(1):73-81, 2012.

[55] Marcin Sabok and Jindřich Zapletal. Forcing properties of ideals of closed sets. J. Symbolic Logic, 76(3):1075-1095, 2011. 
[56] Marion Scheepers. Selection principles and covering properties in topology. Note Mat., 22(2):3-41, 2003.

[57] Saharon Shelah. On cardinal invariants of the continuum. In Axiomatic set theory (Boulder, Colo., 1983), volume 31 of Contemp. Math., pages 183-207. Amer. Math. Soc., Providence, RI, 1984.

[58] Saharon Shelah. Proper and improper forcing. Perspectives in Mathematical Logic. Springer-Verlag, Berlin, second edition, 1998.

[59] Saharon Shelah. On what I do not understand (and have something to say). I. Fund. Math., 166(1-2):1-82, 2000. Saharon Shelah's anniversary issue.

[60] Saharon Shelah. Two cardinal invariants of the continuum $(\mathfrak{d}<\mathfrak{a})$ and FS linearly ordered iterated forcing. Acta Math., 192(2):187-223, 2004.

[61] Asger Törnquist. Definability and almost disjoint families. Adv. Math., 330:61-73, 2018.

[62] Jindřich Zapletal. Forcing idealized, volume 174 of Cambridge Tracts in Mathematics. Cambridge University Press, Cambridge, 2008.

[63] Jindřich Zapletal. Dimension theory and forcing. Topology and its Applications, 167:31 - 35, 2014.

[64] Jindřich Zapletal. Preserving $P$-points in definable forcing. Fund. Math., 204(2):145-154, 2009.

Osvaldo Guzmán

Centro de Ciencias Matematicas, UNAM.

oguzman@matmor.unam.mx

Damjan Kalajdzievski

York University

damjank7354@gmail.com 